\title{
1 Oligomerization-driven MLKL ubiquitylation antagonises necroptosis
}

3 Zikou Liu ${ }^{1,2}$, Laura F. Dagley ${ }^{1,2}$, Kristy Shield-Artin ${ }^{1,2}$, Samuel N. Young ${ }^{1}$, Aleksandra

4 Bankovacki ${ }^{1,6}$, Xiangyi Wang ${ }^{1,2}$, Michelle Tang ${ }^{3,4}$, Jason Howitt ${ }^{3,4}$, Che A. Stafford ${ }^{5}$, Ueli

5 Nachbur $^{1,2}$, Cheree Fitzgibbon ${ }^{1}$, Sarah E. Garnish ${ }^{1,2}$, Andrew I. Webb ${ }^{1,2}$, David Komander ${ }^{1,2}$,

6 James M. Murphy ${ }^{1,2}$, Joanne M. Hildebrand ${ }^{1,2} \wedge$, John Silke ${ }^{1,2} \wedge$

$8{ }^{1}$ The Walter and Eliza Hall Institute of Medical Research, Parkville, VIC 3052, Australia

$9 \quad{ }^{2}$ Department of Medical Biology, University of Melbourne, Parkville, VIC 3052, Australia

$10{ }^{3}$ The Florey Institute of Neuroscience and Mental Health, Parkville, VIC 3052, Australia

$11{ }^{4}$ Swinburne University of Technology, Hawthorn, VIC 3122, Australia

$12{ }^{5}$ Gene Centre and Department of Biochemistry, Ludwig Maximilian University of Munich,

13 Munich 80331, Germany

$14{ }^{6}$ Translational Research, CSL Limited, Melbourne, VIC 3010, Australia

$15 \wedge$ Corresponding authors

\section{Running Title}

18 MLKL ubiquitylation during necroptosis 


\section{Abstract}

2 Mixed lineage kinase domain-like (MLKL) is the executioner in the caspase-independent

3 form of programmed cell death called necroptosis. Receptor Interacting serine/threonine

4 Protein Kinase 3 (RIPK3) phosphorylates MLKL, triggering MLKL oligomerization,

5 membrane translocation and membrane disruption. MLKL also undergoes ubiquitylation

6 during necroptosis, yet neither the mechanism nor significance of this event have been

7 demonstrated. Here we show that necroptosis-specific, multi-mono-ubiquitylation of MLKL

8 occurs following its activation and oligomerization. Ubiquitylated MLKL accumulates in a

9 digitonin insoluble cell fraction comprising plasma/organellar membranes and protein

10 aggregates. This ubiquitylated form is diminished by a plasma membrane located

11 deubiquitylating enzyme. MLKL is ubiquitylated on at least 4 separate lysine residues once

12 oligomerized, and this correlates with proteasome- and lysosome- dependent turnover. Using

13 a MLKL-DUB fusion strategy, we show that constitutive removal of ubiquitin from MLKL

14 licences MLKL auto-activity independent of necroptosis signalling in mouse and human cells.

15 Therefore, besides its role in the kinetic regulation of MLKL-induced death following an

16 exogenous necroptotic stimulus, ubiquitylation also contributes to the restraint of basal levels

17 of activated MLKL to avoid errant cell death.

\section{Key words}

Membranes/MLKL/necroptosis/DUB-fusion/ubiquitylation

\section{Introduction}

23 Necroptosis is a type of programmed cell death that shares some molecular components with

24 the better-known apoptotic cell death pathway, but has distinct morphological features and

25 different physiological consequences. Unlike apoptosis, which is immunologically silent and

26 can be rapidly cleared by neighbouring phagocytic cells (Segawa \& Nagata, 2015),

27 necroptosis induces an inflammatory response by releasing cellular contents including DNA

28 and cytosolic proteins (Kaczmarek et al, 2013). Necroptosis can be induced by a number of

29 stimuli, but is mainly studied downstream of Tumor Necrosis Factor (TNF) ligation to its

30 receptor Tumor Necrosis Factor Receptor 1 (TNFR1). If Inhibitor of APoptosis (IAP)

31 proteins and caspase activities are suppressed, this results in higher order assemblies of

32 Receptor Interacting serine/threonine Protein Kinase 1 (RIPK1) and RIPK3, and subsequent

33 RIPK3 activation and auto-phosphorylation (Sun et al, 2002; Wu et al, 2014). MLKL is 
1 phosphorylated by RIPK3 and oligomerizes, translocates to biological membranes and

2 induces organelle and cell swelling and membrane rupture (Petrie et al, 2019a; Vanden

3 Berghe et al, 2015).

5 Ubiquitylation plays a pivotal role in regulating TNF signalling. In addition to RIPK1

6 ubiquitylation by cIAPs, which forms a platform for MAPK and NF- $\kappa B$ activation (Bertrand

7 et al, 2008), the Linear Ubiquitin Chain Assembly Complex (LUBAC), composed of HOIL-1,

8 HOIP and SHARPIN, generates M1-linked ubiquitin chains on RIPK1, TRADD, TNFR1 and

9 NEMO. This ubiquitylation leads to full activation of IKK and MAPK, and limits TNF

10 induced cell death (Haas et al, 2009; Ikeda et al, 2011; Tokunaga et al, 2009). LUBAC also

11 recruits CYLD, a deubiquitylating enzyme (DUB), that removes M1- and K63-linked

12 ubiquitin chains on RIPK1 and other complex components, while A20 sterically protects M1-

13 linked ubiquitin chains (Dondelinger et al, 2016; Draber et al, 2015).

15 Ubiquitylation has been implicated in the regulation of signalling checkpoints during 16 necroptosis. RIPK3 undergoes K63-linked ubiquitylation on Lys-5, which is believed to 17 support the formation of necrosome. Removal of this ubiquitin chain by the ubiquitin-editing 18 enzyme A20 was proposed to negate necroptosis, because A20 loss led to RIPK3-dependent 19 necroptosis in $\mathrm{T}$ cells and fibroblasts (Onizawa et al, 2015). Furthermore, ubiquitylated 20 TRAF2 was reported to be associated with inactive MLKL, and CYLD deubiquitylates 21 TRAF2 following necroptotic stimulation, allowing TRAF2 to dissociate from MLKL and 22 MLKL to engage with, and be activated by, RIPK3 (Petersen et al, 2015).

24 MLKL has also been shown to undergo ubiquitylation upon necroptotic stimulation 25 (Hildebrand et al, 2020; Lawlor et al, 2015), yet the significance of this post-translational 26 modification and its signalling function are unknown. Here we show that necroptotic 27 signalling stimulates MLKL ubiquitylation and that this antagonises necroptosis via 28 restraining the protein level of activated MLKL. MLKL oligomerization is the crucial stage 29 for MLKL ubiquitylation, but RIPK3 phosphorylation is not necessary because auto-active 30 oligomerizable MLKL mutants are ubiquitylated. MLKL ubiquitylation was removed in vitro 31 by USP21, but not by other chain specific deubiquitylating enzymes, suggesting that MLKL 32 is mono-ubiquitylated at multiples sites. Upon necroptotic stimulation, MLKL variants that 33 are unable to induce cell death can still be ubiquitylated and this correlates with 
1 proteasome/lysosome mediated turnover. Conversely, mutation of four lysine residues 2 identified by mass spectrometry analysis to be ubiquitylated following a necroptotic stimulus 3 did not affect MLKL ubiquitylation or MLKL's cytotoxic activity. We therefore devised a 4 novel approach to completely remove all ubiquitins from MLKL by fusing it to USP21. We 5 showed that this MLKL-USP21 fusion was resistant to ubiquitylation in both human and 6 mouse cell lines and was more cytotoxic when compared with MLKL fused to a catalytically7 inactive USP21. Strikingly, human MLKL, which is very resistant to auto-activation (Petrie 8 et al, 2018; Tanzer et al, 2016), was auto-activated by the USP21 fusion suggesting that 9 ubiquitylation is a major break on the activation of human MLKL. 


\section{Results}

\section{MLKL becomes ubiquitylated during necroptosis}

4 MLKL has previously been reported to undergo ubiquitylation in mouse bone marrow-

5 derived macrophages (BMDMs) stimulated with the necroptotic stimulus: LPS/Smac-

6 mimetic (Compound A)/pan-caspase inhibitor (Q-VD-OPh) (Lawlor et al., 2015). To test

7 whether MLKL ubiquitylation is specific to necroptosis, we stimulated wildtype mouse

8 dermal fibroblasts (MDFs) with TNF (T), Smac-mimetic (S) and the pan-caspase inhibitor

9 IDN-6556 (I) individually or in combination for 3 hrs. GST-UBA immobilised on glutathione

10 sepharose beads (Hospenthal et al, 2015; Stafford et al, 2018) was used to purify

11 ubiquitylated proteins from cell lysates. These purified samples generated a distinct ladder of

12 between 50 and $75 \mathrm{kDa}$ when probed with anti-MLKL, which corresponds to non-

13 ubiquitylated MLKL, mono-ubiquitylated MLKL and multi-ubiquitylated MLKL (Fig. 1A).

14 Ubiquitylated MLKL species were only clearly detected for stimuli that promote MLKL

15 phosphorylation (TI and TSI), but not for stimuli that induce apoptotic cell death (TS) (Fig.

16 1A). Ubiquitylation appears to occur in the same time window as membrane permeabilization,

17 because by the time of UBA-pulldown (UBA-PD) for MDFs (3 hrs) and HT29 cells (16 hrs),

18 cells were $\sim 60 \%$ propidium iodide (PI)-positive (Supp. Fig. 1A).

As expected, TSI failed to induce MLKL ubiquitylation in $T n f r 1^{-/-}$MDFs, but, interestingly, did not completely prevent all TSI induced MLKL phosphorylation (Fig. 1B). Furthermore, the kinase activities of RIPK1 and RIPK3 were required for MLKL ubiquitylation because genetic deletion of Ripk3, or treatment with the RIPK1 and RIPK3 inhibitors, Nec-1 and GSK872, respectively, prevented MLKL ubiquitylation (Fig. 1B). MLKL phosphorylation and ubiquitylation were not, however, reduced by genetic deletion of Traf2 (Fig. 1B). To test whether MLKL ubiquitylation occurred in other cell types, we examined mouse BMDMs. Necroptosis can be induced in BMDMs by a range of different ligands including LPS, Poly I:C and the TNF- receptor superfamily ligand FasL, when they are combined with a Smacmimetic and a caspase inhibitor, IDN-6556 (SI) (Holler et al, 2000; Kaiser et al, 2013). To exclude a role for autocrine TNF, whose synthesis can be induced by a Smac-mimetic, LPS, poly I:C or FasL, we compared BMDMs isolated from $\mathrm{Tnf}^{\prime-}$ and WT mice, and saw the same

32 MLKL ubiquitin laddering pattern in both. This ubiquitylation correlated with MLKL

33 phosphorylation on S345, a well-known hallmark of MLKL activation and necroptosis (Fig. 
1 1C) (Murphy et al, 2013). We and others have shown that human and mouse MLKL are

2 regulated distinctly (Davies et al, 2020; Davies et al, 2018; Li et al, 2015; Petrie et al., 2018;

3 Tanzer et al., 2016), therefore to see whether human MLKL was also ubiquitylated during

4 necroptosis, we treated the human colonic adenocarcinoma HT29 cell line with TSI.

5 Although induction of necroptosis takes longer in HT29 than in MDFs (Supp. Fig. 1B), we

6 observed similar MLKL phosphorylation and ubiquitylation in HT29 cells as in MDFs with

7 similar levels of cell death (Fig. 1D, Supp. Fig. 1B).

9 To determine to what extent upstream necroptotic signalling was required for MLKL 10 ubiquitylation, we used a well-established RIPK3 dimerization strategy to activate RIPK3 in 11 the absence of necroptotic signals (Moujalled et al, 2014; Orozco et al, 2014). We therefore 12 stably expressed a doxycycline-inducible RIPK3-gyrase fusion protein, which can be 13 dimerized and activated by coumermycin (Moujalled et al., 2014), in Ripk3 ${ }^{-}$MDFs. As 14 expected, forced dimerization of RIPK3 induced MLKL phosphorylation but was also able to 15 induce MLKL ubiquitylation independent of TSI stimulation and to similar levels as TSI, 16 indicating that RIPK3 dimerization and activation is sufficient to induce MLKL 17 ubiquitylation (Fig. 1E).

\section{MLKL is ubiquitylated on multiple sites during necroptosis}

20 Ubiquitin can be coupled to, predominantly, lysine residues in target proteins. Ubiquitin itself 21 contains 8 ubiquitylation sites (M1, K6, K11, K27, K29, K33, K48 and K63), which leads to 22 the formation of ubiquitin chains of varying architectures. Differently linked Ub chains serve 23 distinct functions in cells (Swatek \& Komander, 2016). To investigate the ubiquitin architecture on MLKL, we purified ubiquitylated proteins from necroptotic cells using sepharose beads coupled with GST-UBA and digested them on the beads with a set of linkage selective deubiquitylating enzymes (UbiCRest) (Hospenthal et al., 2015). Each DUB specifically recognizes and removes a known subset of poly-ubiquitin chain type with validated activity (Fig. 2A) (Stafford et al., 2018). The non-specific DUB USP21 (Ye et al, 2011) converted MLKL into a non-ubiquitylated form, but the other chain-specific DUBs made no discernible difference to the MLKL ubiquitin laddering pattern (Fig. 2B). To control that there was sufficient DUB activity we decreased the ratio of substrate to DUBs to $1 / 2$ and

32 1/4 (Fig. 2C). vOTU, a DUB that can cleave every type of poly-ubiquitin chain except M133 linked chains, deubiquitylated most ubiquitylated proteins (see total ubiquitin blot, Fig. 2C), 
1 yet it only deubiquitylated the highest molecular weight species of MLKL Ub adducts, and

2 did not affect bands representing the lower molecular weight MLKL Ub species. No changes

3 were observed upon treatment with OTULIN, a DUB specific for M1-linked ubiquitin chains

4 (Keusekotten et al, 2013), indicating that the residual Ub left by vOTU are not M1-linked

5 ubiquitin chains. Given that USP21 is the only DUB in the tested panel that can cleave the

6 covalent bond between a protein substrate and the first ubiquitin unit added, these data

7 suggest that ubiquitylated MLKL is most likely mono-ubiquitylated at multiple sites.

9 MLKL ubiquitylation accumulates in the membrane fraction and can be 10 deubiquitylated by USP21 localised to the plasma membrane

11 During necroptosis, activated MLKL oligomerizes and translocates to biological membranes,

12 ultimately causing membrane rupture and cell death (Cai et al, 2014; Chen et al, 2014;

13 Hildebrand et al, 2014). To understand more about MLKL ubiquitylation, we examined when

14 and where this occurs. WT MDFs were stimulated with TSI over a time course from 30 to

15180 minutes. Cytosolic and crude membrane fractions were generated as previously

16 described using digitonin (Liu et al, 2018), and subjected to UBA-pull down. We refer to the

$170.025 \%$ digitonin insoluble fraction as 'crude membrane', but do not exclude the potential for

18 non-membrane associated large macromolecular/amyloid-like structures to sediment along

19 with crude membranes (Liu et al, 2017). Ubiquitylated MLKL emerged and accumulated

20 within the whole cell lysate and the crude membrane fraction from 90 minutes post-stimulus

21 onwards, coinciding precisely with the appearance of phosphorylated MLKL and onset of

22 cell death (Fig. 3A \& Supp. Fig. 1A). On the other hand, the predominant species of MLKL

23 in the cytosol was non-phosphorylated and non-ubiquitylated (Fig. 3A). These data suggest

24 that MLKL ubiquitylation occurs in the crude membrane fraction but not in the cytosol.

26 To explore further where ubiquitylated MLKL is located we took advantage of the fact that 27 ubiquitylated MLKL was digested by USP21 (Fig. 2B). We therefore fused a CaaX motif tag $28(\mathrm{C}=$ cysteine, $\mathrm{a}=$ aliphatic amino acid, $\mathrm{X}=$ terminal residue) to the $\mathrm{C}$-terminus of the catalytic domain USP21 to localise it to the plasma membrane (Hancock et al, 1991). We chose 'CVLQ' to mimic the CaaX motif found at the C-terminal end of another DUB, USP32

31 (Sapmaz et al, 2019), and also generated a catalytically-dead USP21 mutant version 32 (USP2 $\left.1^{\mathrm{C} 221 \mathrm{R}}\right)$ as a control, also fused with CaaX motif. This mutant, like the previously 33 published mutant USP21 ${ }^{\mathrm{C} 221 \mathrm{~A}}$, lacks the ability to remove ubiquitin (Morrow et al, 2018; Ye 34 et al., 2011; Ye et al, 2012). Using these stably and inducibly expressed constructs we 
1 evaluated TSI-mediated MLKL ubiquitylation with or without USP21-CaaX function. Taking

2 into account the amount of MLKL in the crude membrane fraction it was clear that while it

3 did not completely denude MLKL of ubiquitin, the WT USP21-CaaX fusion did substantially

4 reduce the amount of ubiquitylated MLKL, as well as generally affecting the levels of

5 ubiquitylated proteins (Fig. 3B). Taken together these data suggest that ubiquitylated MLKL

6 is localised to the plasma membrane.

8 Necroptosis induced MLKL ubiquitylation is driven by its oligomerization

9 In order to kill, MLKL oligomerizes, translocates to, and permeabilises membranes. To 10 dissect further the drivers of MLKL ubiquitylation, we generated a series of MLKL mutants 11 that were defective in one or more of these essential steps. Previously, we found that alanine 12 replacement of the surface exposed residues R105 and D106 in the four-helix bundle (4HB) 13 domain of mouse MLKL prevents it from oligomerising, translocating and inducing cell 14 death (Hildebrand et al., 2014; Tanzer et al., 2016). In the context of full length MLKL, we 15 found that these mutations prevent formation of a high molecular weight complex (complex 16 II) in the 'crude membrane' fraction following necroptotic stimulation by TSQ (Fig. 4A). 17 Although R105A/D106A MLKL was phosphorylated following this necroptotic stimulus, it 18 did not exhibit the distinct TSI-induced ubiquitylation observed for WT MLKL (Fig. 4B). 19 This supports the idea that MLKL only undergoes ubiquitylation post activation by RIPK3 20 and suggests that MLKL does not undergo ubiquitylation without oligomerization.

The MLKL mutants, Q343A (which perturbs a hydrogen bond with K219 in the ATP binding motif VTIK) and S345D (phospho-mimetic), are auto-activated forms of murine MLKL (Murphy et al., 2013). We induced expression of Q343A or S345D mutant MLKL in $M l k l^{-/}$ and Ripk $^{-/-} M l k l^{--}$MDFs for $16 \mathrm{hrs}$ where, as predicted, they killed cells independently of an extrinsic necroptotic stimulus (Supp. Fig. 4B). As expected, induction of necroptosis in $M l k l^{--}$cells reconstituted with wild type MLKL resulted in MLKL ubiquitylation (Fig. 4C). However, activated MLKL mutants expressed in the same $M l k l^{--}$MDFs were ubiquitylated independently of a necroptotic stimulus and to an extent comparable to wildtype MLKL induced to undergo necroptosis (Fig. 4C). Furthermore, Q343A MLKL became ubiquitylated in Ripk $^{-/-} \mathrm{Mlkl}^{-/}$MDFs and therefore independently of any upstream activation (Fig. 4D). We conclude that while MLKL ubiquitylation correlates with its oligomerization and

33 phosphorylation, direct RIPK3 association is not required for MLKL ubiquitylation in murine 34 cells. 
2 Necrosulfonamide (NSA) is an inhibitor of human MLKL by forming a covalent bound with

3 Cys86 that only exists in human MLKL but not mouse MLKL (Liu et al., 2017). Consistent

4 with a recent report (Murai et al, 2018; Samson et al, 2020), we found that while it did not

5 inhibit the phosphorylation of MLKL or the formation of high molecular weight MLKL-

6 containing complex, large proportions of this MLKL species remained in the cytosolic $(0.025 \%$

7 digitonin soluble) fraction (C) of cells (Fig. 4E). Consistent with the reduction in MLKL

8 oligomer in the crude membrane $(0.025 \%$ digitonin insoluble) fraction (M), NSA also

9 reduced MLKL ubiquitylation (Fig. 4F).

\section{Ubiquitylated MLKL undergoes proteasome and lysosome dependent turnover}

12 Unlike the R105A/D106A MLKL mutant, E109A/E110A MLKL mutant and N-terminal 13 FLAG-tagged MLKL (N-FLAG MLKL) are able to form higher order oligomers in crude membrane fractions following necroptotic stimulation, yet are nevertheless unable to induce necroptosis (Hildebrand et al., 2014; Tanzer et al., 2016) (Fig. 5, B, Supp. Fig. 5A, B). These mutants therefore allowed us to examine whether ubiquitylation was a consequence of necroptosis. Upon induction of necroptosis, E109A/E110A MLKL was more heavily phosphorylated and ubiquitylated than wildtype MLKL (Fig. 5C). Similarly, N-FLAG MLKL was also phosphorylated and ubiquitylated within 2 hrs of TSI treatment, although these modified MLKL species diminished over time (Fig. 5D).

The cellular turnover of active forms of MLKL has been observed previously by our group and by others (Gong et al, 2017; Hildebrand et al., 2020; Yoon et al, 2017; Zargarian et al, 2017). Therefore, we examined ubiquitylation of N-FLAG MLKL in MDFs over a time course following a TSI pulse. N-FLAG MLKL is phosphorylated, oligomerizes and accumulates in the $0.025 \%$ insoluble cell fraction without killing $M l k l^{-/}$MDFs following necroptotic stimulation. This later feature facilitates the study of these ubiquitylated MLKL species and their cellular turnover in situ using proteasome and lysosome inhibitors as previously described for unmodified MLKL (Hildebrand et al, 2020). We found that after 4 hrs TSI stimulation, levels of ubiquitylated and phosphorylated N-FLAG MLKL dramatically decreased. This decrease can be delayed by either the lysosome inhibitor bafilomycin or the proteasome inhibitor PS341 (Fig. 5E). This suggests that MLKL ubiquitylation correlates 
1 MLKL ubiquitylation antagonises necroptosis

2 We sought to identify the precise amino acids on MLKL that were modified by ubiquitin. We

3 enriched for activated and ubiquitylated N-FLAG MLKL from the 'crude membrane' fraction

4 of MDFs by performing a FLAG tag affinity purification. Eluted fractions from FLAG

5 affinity beads were analysed by mass spectrometry, which identified Gly-Gly conjugates on

6 lysine residues K9, K51, K69 and K77 (Supp. Fig. 6A). All four are located in the 4HB

7 domain of MLKL (Supp. Fig. 6B) which suggests a role for ubiquitylation in regulating

8 MLKL-mediated cell death. We did not identify any additional signs of ubiquitin

9 modification on the brace or pseudokinase region of MLKL using this experimental system.

11 We mutated the four lysines to arginine and inducibly expressed the "4KR" mutant in $M l k l^{-/}$

12 MDFs to explore their role. Surprisingly, the 4KR MLKL was phosphorylated and 13 ubiquitylated in a similar manner to wild type MLKL following TSI treatment (Fig. 6B).

14 There was a reduction in the ability of this mutant to kill cells in response to TSI treatment

15 (Supp. Fig. 6C), but this is seemingly unrelated to its ubiquitylation and more likely due to 16 modification of conserved residues in the cytotoxic domain of MLKL. These results highlight 17 the difficulties in mutational analyses when studying ubiquitylation and rather than mutate 18 any of the other 38 lysines in MLKL we tried a different approach.

We had observed that the deubiquitylating enzyme USP21 removed all ubiquitin from MLKL in vitro (Fig. 2B). We therefore hypothesised that fusing the catalytic domain of USP21 to MLKL would remove TSI induced ubiquitylation from MLKL. We fused it to the C-terminal end of MLKL, because, as with the FLAG tag, N-terminal fusions affect the ability of MLKL to kill (Supp. Fig. 5A). We fused the catalytic domain of USP21 to the C-terminus of both human and mouse MLKL (Fig. 7A) and expressed the fusion proteins in $M L K L^{-/}$HT29 and $M l k l^{-{ }^{-}}$MDF cells respectively, to determine, firstly, whether it still retained cytotoxic activity and, secondly, whether it would be resistant to necroptosis induced ubiquitylation. To specifically control for the loss of ubiquitylation, we also generated MLKL fused to a catalytically-dead USP21 mutant (USP21 ${ }^{\mathrm{C} 221 \mathrm{R}}$; Fig. 7A), as mentioned in Fig. 3B. Both the MLKL-USP21 and MLKL-USP2 $1^{\mathrm{C} 221 \mathrm{R}}$ fusion proteins were exogenously expressed and the cells were stimulated with TSI for varying amounts of time. Ubiquitylated proteins were enriched via UBA-pulldown. Like murine MLKL ${ }^{\mathrm{WT}}$, the catalytically inactive control, MLKL-USP2 ${ }^{\mathrm{C} 221 \mathrm{R}}$, when expressed in $\mathrm{Mlkl}^{-{ }_{-}}$MDFs, showed high MW laddering indicative of ubiquitylation and the ubiquitylation was enhanced following TSI stimulation (Fig. 7B). 
1 These high MW MLKL-USP21 ${ }^{\mathrm{C} 221 \mathrm{R}}$ species were reduced and collapsed into non-

2 ubiquitylated form by digestion of recombinant USP21 (Supp. Fig 7C). In contrast, the 3 ubiquitin laddering was not evident for the MLKL-USP2 ${ }^{\text {WT }}$ fusion (Fig. 7B). MLKL-USP21 4 and MLKL-USP21 ${ }^{\text {C221R }}$ re-constituted sensitivity to TSI stimulation in Mlkl $^{I_{-}}$MDFs with 5 only modestly delayed kinetics when compared to wildtype MLKL (Fig. 7C). However, 6 unlike wildtype MLKL or MLKL-USP21 ${ }^{\mathrm{C} 221 \mathrm{R}}$, MLKL-USP21 could induce cell death 7 independent of TSI stimulation (Fig. 7C), even when expressed in Ripk $3^{-/}$Mlkl ${ }^{-1}$ MDFs (Fig. 8 7C), without being phosphorylated (see Input fractions in Supp. Fig. 7C) .

We made similar observations for human MLKL-USP21 fusions expressed in $M L K L^{-/-}$HT29 cells. Human MLKL-USP21 ${ }^{\mathrm{C} 221 \mathrm{R}}$, but not MLKL-USP21 fusions, became ubiquitylated

12 following TSI stimulation (Fig. 7E). Furthermore, like human MLKL, human MLKL-USP21 and MLKL-USP2 ${ }^{\mathrm{C} 221 \mathrm{R}}$ fusions were able to reconstitute the capacity of $M L K L^{-/}$HT29 cells to undergo TSI induced necroptosis when their expression was induced by doxycycline (Fig. 7D), while USP21 controls did not (Supp. Fig. 7B). For the first $10 \mathrm{hrs}$ of expression and activation, NSA blocked TSI-induced cell death for all MLKL species, which was then overcome by overexpression. MLKL-USP21 also exhibited TSI-independent cell death, occurring from $16 \mathrm{hrs}$, which can also be delayed by NSA. (Fig. 7D). While phosphorylated MLKL was not detected by that time (Fig. 7E). These data indicate that ubiquitylation of MLKL is not required for necroptotic cell death, but rather acts as an important kinetic regulator of the necroptosis pathway following oligomerization and membrane association.

\section{Discussion}

MLKL undergoes ubiquitylation during necroptosis. In this study, we identified distinctive ubiquitylation of both mouse and human MLKL that could be induced by a range of necroptotic stimuli. This signature ubiquitin ladder was essentially resistant to cleavage by a host of DUBs. Only USP21, the DUB that can remove all ubiquitin modifications, including mono-ubiquitylation, was able to deubiquitylate MLKL. Therefore, we propose that MLKL is mono-ubiquitylated at multiple sites. Mono-ubiquitylation is typically associated with endosome-lysosome trafficking (Haglund et al, 2003; Mosesson \& Yarden, 2006) and is less

32 likely to play a scaffolding role or result in proteasomal degradation (Oh et al, 2018;

33 Wilkinson et al, 1995). 
2 Full activation of MLKL's killing activity is a multi-step and protracted process, the

3 choreography of which has not yet been fully deduced. Nevertheless there is a broad

4 consensus, based on analysis of MLKL mutants and a range of different techniques, that

5 phosphorylation by RIPK3 leads to MLKL oligomerization, translocation to biological

6 membranes and subsequent membrane permeabilization (Petrie et al, 2020; Petrie et al., 2018;

7 Samson et al., 2020; Tanzer et al., 2016). Recent single-cell imaging approaches examining

8 human MLKL show that phosphorylated MLKL clusters in cytoplasmic vesicles together

9 with RIPK1. These vesicles are actively transported to the plasma membrane, where they

10 begin to coalesce in hotspots, some hours prior to membrane lysis (Samson et al., 2020).

11 Consistent with data showing that NSA does not prevent MLKL phosphorylation or initial

12 oligomerization, but does block higher order oligomerization (Liu et al., 2017)), NSA

13 appears to prevent MLKL clustering (Samson et al., 2020). To define where and when

14 ubiquitylation of MLKL occurred we fractionated cells into cytosolic and crude membrane

15 fractions, and only detected ubiquitylated MLKL in the $0.025 \%$ digitonin insoluble (crude

16 membrane) fraction. We also examined a number of different MLKL mutants that we had

17 previously characterised for their ability to form oligomers, translocate to the crude

18 membrane fraction and promote necroptosis. The MLKL mutants, Q343A and S345D, which

19 are able to form $0.025 \%$ digitonin insoluble high molecular weight oligomers and induce cell

20 death without necroptotic stimulation (Murphy et al., 2013; Tanzer et al, 2015), were

21 ubiquitylated independently of RIPK3 or a necroptotic TSQ stimulus. On the other hand, the

$22 \mathrm{R} 105 \mathrm{~A} / \mathrm{D} 106 \mathrm{~A}$ mutant that is unable to form high molecular weight oligomers on BN-PAGE

23 did not become ubiquitylated following a necroptotic stimulus. This implies that MLKL

24 ubiquitylation occurs after MLKL oligomerization and does not specifically require RIPK3 or

25 upstream signalling.

27 MLKL has been variously reported to traffic to the nucleus (Weber et al, 2018; Yoon et al, 28 2016), lysosomes (Fan et al, 2019; Wang et al, 2014; Yoon et al., 2017), mitochondria 29 (Wang et al, 2012) and the plasma membrane (the most likely location) (Cai et al., 2014;

30 Chen et al., 2014; Hildebrand et al., 2014; Samson et al., 2020) following necroptotic 31 activation. Crude membrane fractionation enriches membrane and insoluble protein 32 aggregates, therefore in an attempt to determine more precisely where MLKL ubiquitylation 33 occurred, we generated a plasma membrane-targeted, CaaX-fused USP21 (Wright \& Philips, 34 2006). Because ubiquitin modification plays a role in the initial, plasma membrane located, 
1 TNF/TNFR1 induced necroptotic signalling, we anticipated that expressing USP21-CaaX

2 might affect induction of necroptosis. However necroptosis was induced in cells expressing a 3 non-targeted USP21 or a catalytically inactive USP $21^{\mathrm{C} 221 \mathrm{R}}-\mathrm{CaaX}$ to equal levels as in

4 USP21-CaaX cells, despite the fact that USP21-CaaX was clearly active and reduced total

5 ubiquitin levels. Consistent with MLKL being targeted to the plasma membrane, MLKL was

6 less ubiquitylated in USP21-CaaX cells than in cells expressing USP21 ${ }^{\mathrm{C} 221 \mathrm{R}}$-CaaX following

7 a necroptotic stimulus. MLKL ubiquitylation was, however, not completely prevented by

8 USP21-CaaX expression leaving open several interpretations of this data viz: not all MLKL

9 is ubiquitylated at the plasma membrane; or USP21-CaaX was unable to access all MLKL in

10 the membrane; or MLKL is ubiquitylated prior to reaching the plasma membrane and can

11 only be deubiquitylated once it arrives there. Therefore while we cannot exclude that

12 ubiquitylated MLKL sediments as part of large amyloid-like polymers or large cytoskeletal

13 structures in the crude membrane fraction without being directly associated with a biological

14 membrane (Liu et al., 2017), our data suggest that at least some ubiquitylated MLKL is

15 accessible to a plasma membrane localised DUB.

As previously observed (Murai et al., 2018), we found that in the presence of NSA, the high molecular weight human MLKL complex that is normally found exclusively in the crude membrane fraction was now also observed in the cytosolic fraction. Combined with other observations (Samson et al., 2020), this indicates that NSA interferes with MLKL membrane association. Since NSA treatment also inhibited MLKL-ubiquitylation, this suggests that membrane association is required for MLKL ubiquitylation, and that the relevant E3 ligase is also membrane associated.

Neither N-terminally FLAG tagged MLKL nor the E109A/E110A mutant MLKL are capable of killing cells, although both are phosphorylated and form $0.025 \%$ digitonin-insoluble high molecular weight oligomers in a similar manner to wild type MLKL following induction of necroptosis. Since both are also ubiquitylated we can conclude that ubiquitylation is not sufficient to cause necroptosis. To more precisely define the role of MLKL ubiquitylation during necroptosis we used mass spectrometry to identify ubiquitylated lysines in mouse MLKL. We found four such lysines in the 4HB domain, but none in the pseudokinase domain. Only one of these four, K77, is conserved in human MLKL, however this is not particularly

33 surprising because homology of charged residues in MLKL is not well conserved between mouse and human (Supp. Fig. 6B). 
2 Mutation of these lysines did not prevent either MLKL induced killing or MLKL

3 ubiquitylation. The persistence of ubiquitin modification is a relatively common scenario

4 when trying to define the role of ubiquitylation by mutating lysine. This is because E3 ligase

5 mediated ubiquitylation is rarely tightly restricted to a motif and can therefore be

6 promiscuous in target modification (Petroski \& Deshaies, 2003; Wu et al, 2003). This means

7 that if a favoured lysine in the target is mutated, the E3 ligase may nevertheless ubiquitylate

8 another lysine. We therefore tried a more innovative approach. Since we had shown that the

9 DUB USP21 was able to remove necroptosis induced ubiquitylation of MLKL in vitro, we

10 generated an MLKL C-terminal USP21 fusion that we predicted would be constitutively

11 deubiquitylated. A similar approach fusing the K63 specific DUB AMSH to EGFR has been

12 used to investigate EGFR degradation (Huang et al, 2013), however it was not clear whether

13 a DUB that removes all ubiquitin would be as well tolerated by cells. Stable inducible

14 expression of USP21 alone was not toxic to cells over 24 hours and fusion of the wildtype or

15 catalytically-dead USP21 did not markedly affect MLKL's cytotoxic activity following a TSI

16 death stimulus. Furthermore, catalytically-active USP21 fused to MLKL prevented TSI

17 induced ubiquitylation of MLKL. Interestingly, in both human and mouse cells, loss of

18 MLKL ubiquitylation also allowed MLKL to kill cells without a necroptotic stimulus, albeit

19 less potently when compared with such a stimulus. This further supports the idea that

20 ubiquitylation of MLKL is an important 'insurance policy' against low level activation of

21 MLKL by other cellular kinases or low level spontaneous transition to the active

22 conformation. Furthermore our results indicate that the approach of directly fusing a DUB,

23 even a pan DUB like USP21, to a protein of interest may be a widely applicable technique to

24 evaluate the role of ubiquitin modification in other systems.

26 Other mechanisms have been proposed for MLKL turnover post-activation. The ESCRT-III

27 machinery was proposed to mediate plasma membrane shedding alongside active MLKL

28 during necroptosis (Gong et al., 2017), and cells were reported to release active MLKL

29 containing vesicles via endosomal trafficking (Fan et al., 2019; Yoon et al., 2017; Zargarian

30 et al., 2017). It has been proposed that this turnover helps set a threshold of activated MLKL

31 required for necroptosis and that it delays the onset of cell death to allow production of

32 essential cytokines and Damage-associated molecular patterns (DAMPs) and an

33 inflammatory response (Vandenabeele et al, 2017). Another possibility is that the prolonged

34 multi-step path from MLKL phosphorylation to membrane permeabilization and cell death 
1 allows multiple points of regulation that ensure that a cell only commits to necroptosis under

2 precisely defined conditions (Dovey et al, 2018; Hildebrand et al., 2020; Jacobsen et al, 2016;

3 Samson et al., 2020). For this to work, the cell must have mechanisms to deactivate already

4 activated MLKL, and the ubiquitylation of MLKL may serve as one such mechanism. Our

5 results, showing that MLKL-USP21, but not a wild type MLKL expressed to similar levels,

6 kills cells in the absence of a necroptotic stimulus suggest that MLKL may be continually

7 translocating to membranes and turned over by MLKL-ubiquitylation. Like the rapid

8 degradation of inflammatory cytokine mRNAs (Lacey et al, 2015; Menon \& Gaestel, 2018)

9 although energetically costly, this might allow a more rapid response to a pathogen than an

10 on/off mechanism focused solely on initiation and may also provide another mechanism to

11 detect and act on attempts by pathogens to interfere with this anti-pathogen response.

\section{Materials and Methods}

\section{Compounds and cytokines}

16 Human TNF-Fc made in-house, Smac-mimetics Compound A (Tetralogic), IDN-6556

17 (Tetralogic), Q-VD-OPh (R \& D Systems), Lipopolysaccharide (LPS, Sigma), Poly I:C 18 (Sigma), Fas Ligand (a gift from Lorraine O'Reilly, WEHI), doxycycline (Sigma), 19 Necrostatin-1 (Nec-1, Sigma), GSK872 (a gift from Anaxis Pty, Ltd.), coumermycin (Sigma), 20 Necrosulfonamide (NSA, Merck Millipore), Propidium iodide (Sigma), Sytox Green 21 (Thermo Fisher), puromycin (Thermo Fisher), N-ethylmaleimide (NEM, Sigma), 22 deubiquitylase (DUBs) made in house (Hospenthal et al., 2015), complete protease inhibitor 23 cocktail (Roche), Bafilomycin A1 (BAF, Enzo), PS341 (Sigma).

\section{Antibodies:}

26 Mouse phospho-MLKL (Ser345) rabbit monoclonal ERP9515(2) (Abcam)

27 Mouse phospho-MLKL (Ser345) rabbit monoclonal (D6E3G) (Cell Signalling Technology)

28 MLKL rat monoclonal 3H1 (available from Millipore MABC604; generated in-house)

$29 \quad \beta$-actin mouse monoclonal AC-15 (Sigma A-1978)

30 BAK NT rabbit polyclonal \#06-536 (EMD Millipore)

31 GAPDH rabbit monoclonal 14C10 (Cell Signalling Technology)

32 Pan-ubiquitin mouse monoclonal \#3939 (Cell Signalling Technology)

33 Mouse/human RIPK1 mouse monoclonal \#610458 (BD transduction Laboratories) 
1 Mouse RIPK3 rabbit polyclonal PSC-2283-c100 (Axxora (Pro Sci))

2 Human RIPK3 rat monoclonal 1H2 (generated in-house)(Petrie et al, 2019b)

3 Human phospho-MLKL (Ser358) rabbit monoclonal ab 187091 (Abcam)

$4 \quad$ VDAC1 rabbit polyclonal AB10527 (Millipore)

5 Human Usp21 rabbit polyclonal 17856-I-AP (Proteintech)

6 HRP-conjugated secondary antibodies

\section{Cell lines immortalization and transfection}

9 Cells were cultured in DMEM $+8 \%$ FCS at $37^{\circ} \mathrm{C}$. HT29 cells were a kind gift from Mark

10 Hampton. MDFs were isolated from tails of mice bearing different genotype, and 11 immortalized by SV40 large T antigen via lentivirus transduction. MLKL mutant constructs 12 were generated as described previously ((Hildebrand et al., 2014; Moujalled et al., 2014;

13 Murphy et al., 2013)). All oligonucleotides for PCR and mutagenesis were synthesized by

14 IDT. Constructs encoding USP21 catalytic domain with GS linker were made by GenScript 15 (Nanjing, CN). Genes encoding WT mouse MLKL and PCR-derived mutants were cloned 16 into $\mathrm{pF}$ TRE3G PGK puro, a puromycin-selectable, doxycycline-inducible vector as 17 previously described (and kindly supplied by Toru Okamoto) (Hildebrand et al., 2014; 18 Moujalled et al., 2014; Murphy et al., 2013; Tanzer et al., 2016). Lentiviruses were generated 19 in HEK293T cells before infection of target cells. Puromycin $(5 \mu \mathrm{g} / \mathrm{mL})$ was added for 20 selection and maintenance of lines stably transduced with lentivirus.

22 For BMDMs, femora and tibiae were collected from WT and $T n f^{/-}$mice at 6 weeks old, cut 23 and flushed by PBS. Bone marrow were incubated in Petri dishes with DMEM supplemented 24 with 10\% FCS and 20\% conditioned L929 medium for 7 days. Cells were fed with fresh medium after 3 days of plating.

\section{Concentrations of stimuli and inhibitors}

28 TNF (100 ng/mL), Smac-mimetic Compound A (500 nM), IDN-6556 (5 $\mu \mathrm{M})$, Q-VD-OPh (5 $29 \mu \mathrm{M})$, doxycycline $(20 \mathrm{ng} / \mathrm{mL})$, Necrostatin-1 (50 $\mu \mathrm{M})$, GSK872 (5 mM), LPS $(50 \mathrm{ng} / \mathrm{mL})$, 30 Fas ligand $(6.4 \mathrm{ng} / \mathrm{mL})$, Poly I:C (1 mg/mL), coumermycin $(700 \mathrm{nM})$, Bafilomycin $(5 \mu \mathrm{M})$, 31 PS341 (50 nM), MG132 (200nM), Chloroquine (50 $\mu \mathrm{M}), \mathrm{NH}_{4} \mathrm{Cl}(2 \mathrm{mM}), \mathrm{Ca}-074 \mathrm{Me}(20$ $32 \mu \mathrm{M})$, TLCK $(100 \mu \mathrm{M}), \operatorname{AEBSF}(100 \mu \mathrm{M})$ and concentrations otherwise indicated. 


\section{Cell death measured by flow cytometry}

2 Cells were plated at 50,000/well in 24-well plates before treatment, collected by trypsin and

3 spun down, resuspended and stained with PI (Propidium Iodide, $1 \mu \mathrm{g} / \mathrm{mL}$ ) in PBS buffer, and

4 quantified by Flow cytometry.

6 Cell death measured by live cell imaging

7 MDFs were plates at 10,000/well or 15,000/well (96-well plates) and HT29s were plated at 8 40,000/well (48-well plates) or 15,000/well (96-well plates) and allowed to settle for 4 hrs 9 (MDFs) and $24 \mathrm{hrs}$ (HT29s) respectively. Cells were stimulated as indicated in media 10 containing Propidium Iodide (PI, 200 ng/mL) or Sytox Green (500nM, ThermoFisher 11 Scientific) and imaged at $45 \mathrm{~min}$ or $1 \mathrm{hr}$ intervals using an IncuCyte S3 Live cell imager.

12 Numbers of PI or Sytox positive cells were quantified and plotted using IncuCyte software.

\section{Cellular fractionation and Blue Native-PAGE}

15 Cells were collected by scraping, spun down and washed in pre-chilled PBS. MELB buffer 16 (20 mM 4-(2-Hydroxyethyl) piperazine-1-ethanesulfonic acid, (HEPES) pH=7.5, $100 \mathrm{mM}$ 17 sucrose, $2.5 \mathrm{mM} \mathrm{MgCl} 2,100 \mathrm{mM} \mathrm{KCl}$ ) containing $0.025 \%$ digitonin was used to 18 permeabilise cells to extract the cytosol fraction. Non-soluble part was further solubilized by $191 \%$ digitonin buffer. (Liu et al., 2018; Schagger \& von Jagow, 1991) Both fractions were 20 separated by Bis-Tris Native PAGE, and transferred to PVDF membrane. After transfer the 21 membrane was then destained (50\% methanol and $25 \%$ acetic acid) and denatured (in $6 \mathrm{M}$ 22 guanidine $\mathrm{HCl}, 5 \mathrm{mM} \beta-\mathrm{ME})$ to maximise epitope exposure.

\section{UBA-pull down assay and UbiCRest}

25 Recombinant GST-UBA fusion protein (Hjerpe et al, 2009) purified from E. coli in house 26 was prebound to glutathione sepharose beads (10 $\mu \mathrm{L} /$ condition). Cells were lysed in DISC 27 buffer (30 mM Tris- $\mathrm{HCl}, \mathrm{pH} 7.5,150 \mathrm{mM} \mathrm{NaCl}, 10 \%$ glycerol) containing 1\% Triton X-100 28 with $10 \mathrm{mM}$ NEM and protease inhibitor. Cleared lysate was then coupled with beads 29 overnight. SDS-PAGE sample loading buffer was used to elute the UBA-pull down fraction.

30 For UbiCrest, washed beads were then incubated with DUBs at previously established 31 concentrations (Hospenthal et al., 2015) and incubated at $37^{\circ} \mathrm{C}$ for the indicated times. 32 Digestion product was eluted in SDS sample buffer for Western blot analysis. Beads elution 
1 fractions were generated by removing the digestion product from the beads and following

2 washing.

4 FLAG-tagged protein immunoaffinity precipitation and Mass Spectrometry sample 5 preparation

6 N-FLAG WT mouse MLKL was inducibly expressed in $M l k l^{--}$MDFs by doxycycline 7 overnight and stimulated with TSI for 3 hrs. Cells were collected by scraping and 8 permeabilised by $0.025 \%$ digitonin in MELB buffer together with NEM (10 mM) and 9 protease inhibitors. The crude membrane fraction was pelleted by centrifugation and 10 dissolved in $1 \%$ SDS in DISC buffer. Cleared lysate of crude membrane was then applied to 11 M2-FLAG beads for affinity precipitation. FLAG tagged protein on beads was eluted by 12 heating at $56^{\circ} \mathrm{C}$ in $0.5 \%$ SDS solution. Eluted material was subjected to tryptic digestion 13 using the FASP method (Wisniewski et al, 2009). Peptides were lyophilised using CentriVap 14 (Labconco) prior to reconstituting in $80 \mu \mathrm{l} 0.1 \%$ FA/2\% acetonitrile (ACN). Peptide mixtures $15(1 \mu \mathrm{l})$ were analysed by nanoflow reversed-phase liquid chromatography tandem mass spectrometry (LC-MS/MS) on an M-Class HPLC (Waters) coupled to a Q-Exactive Orbitrap mass spectrometer (Thermo Fisher). Peptide mixtures were loaded in buffer A $(0.1 \%$ formic acid, 2\% acetonitrile, Milli-Q water), and separated by reverse-phase chromatography using

$19 \mathrm{C}_{18}$ fused silica column (packed emitter, I.D. $75 \mu \mathrm{m}$, O.D. $360 \mu \mathrm{m}$ x $25 \mathrm{~cm}$ length, 20 IonOpticks, Australia) using flow rates and data-dependent methods as previously described 21 (Delconte et al, 2016; Kedzierski et al, 2017). Raw files consisting of high-resolution 22 MS/MS spectra were processed with MaxQuant (version 1.5.8.3) for feature detection and 23 protein identification using the Andromeda search engine (Cox et al, 2011). Extracted peak 24 lists were searched against the UniProtKB/Swiss-Prot Mus musculus database (October 2016) 25 and a separate reverse decoy database to empirically assess the false discovery rate (FDR) 26 using strict trypsin specificity allowing up to 2 missed cleavages. The minimum required 27 peptide length was set to 7 amino acids. The mass tolerance for precursor ions and fragment 28 ions were $20 \mathrm{ppm}$ and $0.5 \mathrm{Da}$, respectively. The search included variable modifications of 29 oxidation (methionine), amino-terminal acetylation, carbamidomethyl (cysteine), GlyGly or 30 ubiquitylation (lysine), phosphorylation (serine, threonine or tyrosine) and N-ethylmaleimide 31 (cysteine).

\section{Data availability}


1 The raw mass spectrometric data and the MaxQuant result files have been deposited to the

2 ProteomeXchange Consortium via the PRIDE (Perez-Riverol et al, 2019) partner repository

3 with the dataset identifier: PXD015537.

4 Username: reviewer27536@ ebi.ac.uk Password: f2WTYnC4

5

6 Acknowledgements:

7 We would like to thank Jiami Han, Yueyuan Li and the WEHI mouse facility for technical 8 assistance. This work was funded by NHMRC grants 1025594 (JS), 1046984 (JS) and 91105023 (JS and JMH) and fellowships 1172929 (JMM), 1058190 (JS), 1107149 (JS) and 10110574 (JS) and was made possible through Victorian State Government Operational 11 Infrastructure Support and Australian Government NHMRC IRIISS (9000587).

\section{Author Contribution}

14 ZL, LD and KSA designed and performed experiments, and analysed data. ZL, JMH and JS

15 analysed the data and wrote the manuscript. DK conceived the DUB fusion experiment and 16 provided reagents. All authors read and commented on the manuscript.

\section{Conflict of interest statement}

19 SNY, AB, UN, CF, SEG, JMM, JMH and JS contribute to, or have contributed to, a project 20 with Anaxis Pty Ltd to develop necroptosis inhibitors. 


\section{Figure legends}

3 Abbreviations: TNF (T), Smac-mimetics Compound A (S), IDN-6556 (I), Q-VD-OPh (Q),

4 whole cell lysate (WCL), GST-UBA pull down fractions (UBA-PD), cytosolic fraction (C),

5 crude membrane fraction (M), wildtype (WT), C221R (CR), Propidium Iodide (PI),

6 Necrostatin-1 (Nec-1), ubiquitin (Ub) and others as indicated elsewhere. TS, SI, TSI and TSQ

7 are used in combination, as apoptotic or necroptotic stimuli.

\section{Figure 1. MLKL undergoes ubiquitylation during necroptosis}

11 A WT MDFs were treated \pm TSI individually or in combination for 3 hrs. Whole cell lysates

12 (WCL) and UBA-pull down (UBA-PD) fractions were analysed by Western blot and 13 probed with antibodies as indicated. Representative of three independent experiments. 14 Samples of UBA-pull down in following figures were analysed in the same way unless 15 otherwise indicated.

16 B WT, Ripk3 ${ }^{-/}, M_{k l} l^{-/}, \operatorname{Tnfr} 1^{-/}$and Traf2 $2^{-/}$MDFs were untreated (UT) or treated with TSI 17 for 3 hrs. Nec-1 and GSK872 were added to inhibit RIPK1 and RIPK3 kinase activities 18 respectively.

$19 \mathrm{C}$ WT and $T n f^{\prime-}$ BMDMs were treated \pm death ligands including TNF (T), LPS (L), Fas 20 ligand (F) and Poly I:C (P) in addition to $\mathrm{S}$ and $\mathrm{I}$ for $3 \mathrm{hrs}$.

21 D WT HT29 cells were untreated (UT) or treated with TSI for $16 \mathrm{hrs.}$

22 E RIPK3-gyrase were inducibly expressed in Ripk $3^{-/-}$MDFs by doxycycline (dox) for $5 \mathrm{hrs,}$ 23 and cells were then treated \pm combination of TSI, or coumermycin (coum) for $3 \mathrm{hrs}$.

Supplementary Figure 1. Cell death time course of MDFs and HT29 cells following necroptotic stimulation

MDFs (A) and HT29 (B) cells were treated with TSI. Cell death was measured by PI staining 
1 Figure 2. MLKL is mono-ubiquitylated at multiple sites

3 A Deubiquitylating enzymes (DUBs) and their ubiquitin substrates. Less efficiently cleaved substrates are indicated in brackets.

5 B UBA-pull down from WT MDFs treated with TSI for $3 \mathrm{hrs}$ were subjected to the DUBs shown in (A). Beads eluates were analysed by Western blot and probed with antibodies as indicated. Representative of three independent experiments.

8 C $1.4 \mathrm{~mL}$ cleared cell lysate from $4 \times 10^{6}$ TSI-treated WT MDFs was split into three parts of 9 the indicated volume, followed by UBA-pull down and DUB incubation. Bead eluates were analysed by Western blot and probed with the indicated antibodies. Representative of

Figure 3. MLKL ubiquitylation accumulates in the crude membrane fraction and can be digested by USP21 located on biological membranes.

A WT MDFs were treated with TSI for indicated time. Cells were fractionated into cytosol and crude membrane parts, followed by UBA-pull down. All fractions were analysed by Western blot and probed with antibodies as indicated. Representative of three independent experiments.

B WT USP21-CaaX and USP21 ${ }^{\mathrm{C} 221 \mathrm{R}}$-CaaX (CR) were inducibly expressed in WT MDFs by doxycycline for $5 \mathrm{hrs}$. Ubiquitylated proteins were enriched followed by TSI stimulation and cellular fractionation. All fractions were analysed by Western blot and probed with antibodies as indicated. Representative of three independent experiments.

\section{Supplementary Figure 3. USP21-CaaX expression does not alter the kinetics of TNF} induced apoptosis or necroptosis in MDFs.

A WT USP21, USP21-CaaX and USP21 ${ }^{\mathrm{C} 221 \mathrm{R}}$-CaaX were inducibly expressed in WT MDFs by doxycycline. TS and Nec-1 were used to control for apoptotic signalling. Sytox Green positive cells were quantified in real time by live cell imaging. Representative of 2 independent experiments. 
1 Figure 4. MLKL oligomerization drives its necroptosis specific ubiquitylation 2

3 A WT and R105AD106A mutant MLKL were inducibly expressed in $M l k l^{-\alpha}$ MDFs by 4 doxycycline, at the same time cells were untreated (UT) or treated with for $6 \mathrm{hrs}$. Cells

5 were fractionated into cytosol (C) and crude membrane (M). Fractions were analysed by

6 BN- or SDS-PAGE, Western blot and probed with the indicated antibodies.

7 Representative of three independent experiments.

8 B Cell lysates from (A) were subjected to UBA-pull down and analysed as described above.

9 C WT, Q343A and S345D mutant MLKL were inducibly expressed in $\mathrm{Mlkl}^{-/}$MDFs by 10 doxycycline, at the same time cells were treated \pm TSQ for $16 \mathrm{hrs}$, followed by UBA-

11 pulldown. Representative of three independent experiments.

12 D WT and Q343A mutant MLKL were inducibly expressed in Ripk3 $^{-/} \mathrm{Mlkl}^{-/}$MDFs by 13 doxycycline, at the same time cells were treated \pm TSQ for $16 \mathrm{hrs}$, followed by UBA14 pulldown. Representative of three independent experiments.

15 E HT29 cells were stimulated with TSI, \pm NSA (500 nM), or left untreated (UT) for 16 hrs, 16 followed by cellular fractionation. Fractions were analysed by BN- or SDS-PAGE, 17 Western blot and probed with the indicated antibodies. Representative of three 18 independent experiments.

19 F Cell lysates from (E) were subjected to UBA-pulldown and analysed as described above.

21 Supplementary Figure 4. MLKL oligomerization drives its necroptosis specific 22 ubiquitylation

A Cell death of samples from Fig. 4A was measured by PI staining based on flow cytometry. Data are plotted as mean \pm SEM of three independent experiments.

26 B Cell death of samples from Fig. 4C, D were analysed as in (A). 
1 Figure 5. MLKL-ubiquitylation does not lead to cell death, but correlates with the turnover of activated MLKL

A WT and E109AE110A mutant MLKL were inducibly expressed in $M l k l^{--}$MDFs by doxycycline, cells were untreated (UT) or treated with TSQ for 6 hrs (please note that this same control was used in Figure 4A). Cell death was measured by PI staining based on flow cytometry. Data are plotted as mean \pm SEM of three independent experiments.

B Cellular fractions from (A) were analysed by Western blot from BN-PAGE or SDS-PAGE using antibodies as indicated. Representative of three independent experiments.

C Cell lysates from (A) were subjected to UBA-pull down and analysed as described above.

D N-FLAG MLKL were inducibly expressed in $M l k l^{--}$MDFs by doxycycline overnight, and

\section{Supplementary Figure 5. N-FLAG MLKL behaves like WT MLKL but does not induce}

\section{cell death following necroptotic stimulation}

A WT MLKL and N-FLAG MLKL were inducibly expressed in $M l k l^{-/}$MDFs by doxycycline for $12 \mathrm{hrs}$ and cells were treated with the TSI or TSQ necroptotic stimuli. TS was included to control for responding to apoptosis signalling. Cell death was measured by PI staining based on flow cytometry. Data are plotted as mean \pm SEM of three independent experiments.

B WT MLKL and N-FLAG MLKL were inducibly expressed in $M l k l^{-/}$MDFs by doxycycline for $6 \mathrm{hrs}$, cells were untreated (UT) or treated with TSQ. Cellular fractions were analysed by Western blot from BN-PAGE or SDS-PAGE using antibodies as indicated. Representative of three independent experiments. 
1 Figure 6. Simultaneous arginine replacement of 4 ubiquitylation sites on the mMLKL

2 4HB domain does not prevent necroptosis-induced ubiquitylation.

4 A Cartoon of the N-terminal region (residues 1-180) of mouse MLKL (PDB accession 4BTF;(Murphy et al., 2013)) showing the four lysine residues identified from MS analysis as yellow sticks.

7 B WT and 4KR mutant MLKL were inducibly expressed in $\mathrm{Mlkl}^{-/}$MDFs by doxycycline for $86 \mathrm{hrs}$ and cells were untreated (UT) or treated with TSI, followed by UBA-pull down. 9 Representative of three independent experiments.

Supplementary Figure 6. Simultaneous arginine replacement of 4 ubiquitylation sites on

A MS spectra were manually validated to confirm the identification of four Gly-Gly sites on activated MLKL.

B Alignment of mouse and human MLKL N-terminal domain. Positively charged residues are labelled in blue and negatively charged residues are labelled in pink.

$\mathrm{C}$ WT and 4KR mutant MLKL were inducibly expressed in $\mathrm{Mlkl}^{-/} \mathrm{MDFs}$ by doxycycline and cells were treated \pm TSI (added simultaneously) for 4 hrs. Sytox Green positive cells were quantified in real time by IncuCyte S3 live cell imaging. Representative of 3 independent

Figure 7. MLKL ubiquitylation antagonises necroptosis

A Schematic of the MLKL-USP21 and USP21 catalytic dead mutant proteins.

B Mouse MLKL-USP21 and MLKL-USP21 ${ }^{\mathrm{C} 221 \mathrm{R}}$ were inducibly expressed in $\mathrm{Mlkl}^{--}$MDFs by doxycycline $(10 \mathrm{ng} / \mathrm{mL})$ for $6 \mathrm{hrs}$ with addition of a necroptotic stimulus (TSI) for indicated time, followed by UBA-pulldown. Representative of three independent experiments.

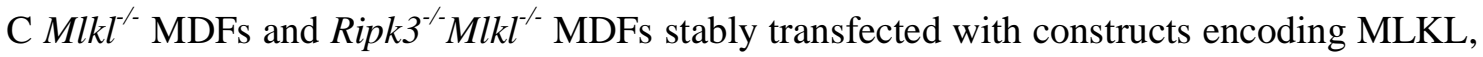
USP21, USP21 ${ }^{\mathrm{C} 221 \mathrm{R}}$, MLKL-USP21 and MLKL-USP21 ${ }^{\mathrm{C} 221 \mathrm{R}}$ were treated with doxycycline, TSI or in combination. Propidium iodide positive cells were quantified in real time by IncuCyte live cell imaging. Representative of three independent experiments. 
1 D $M L K L^{-/-}$HT29 cells stably transfected with constructs encoding human MLKL-USP21 and 2 MLKL-USP2 $1^{\mathrm{C} 221 \mathrm{R}}$, were treated with doxycycline, NSA $(1 \mu \mathrm{M})$, TSI or combinations 3 thereof (added simultaneously). Sytox Green positive cells were quantified in real time by 4 live cell imaging. Representative of 6 independent experiments. (A red dashed line is 5 shown to highlight the delay in death kinetics upon treatment with NSA).

6 E Human MLKL-USP21 and MLKL-USP21 ${ }^{\mathrm{C} 221 \mathrm{R}}$ were inducibly expressed in $M L K L^{-/}$ 7 HT29 cells by doxycycline $(10 \mathrm{ng} / \mathrm{mL})$ for $16 \mathrm{hrs}$ and then cells were treated with TSI for 8 the indicated time course, followed by UBA-pulldown. Representative of three 9 independent experiments.

\section{Supplementary Figure 7. MLKL ubiquitylation antagonises necroptosis}

A Mouse MLKL, human USP21 and mouse MLKL-human USP21 fusions were inducibly expressed in Ripk3 $3^{-/} M l k l^{--}$MDFs following doxycycline addition $(20 \mathrm{ng} / \mathrm{mL})$ for 6 hours \pm TSI. Representative of 2 independent experiments.

B $M L K L^{-/}$HT29 cells stably transfected with doxycycline inducible constructs encoding human USP2 1 and human USP2 $1^{\text {C221R }}$ were treated with doxycycline, NSA $(1 \mu \mathrm{M})$, TSI or combinations thereof (added simultaneously). Sytox Green positive cells were quantified in real time by live cell imaging. Representative of 2 independent experiments.

C Mouse MLKL-USP21 and MLKL-USP21 ${ }^{\mathrm{C} 221 \mathrm{R}}$ were inducibly expressed in $\mathrm{Mlkl}^{-\mathrm{MDF}^{-}}$MDs by doxycycline $(10 \mathrm{ng} / \mathrm{mL})$ for $8 \mathrm{hrs}$ with addition of a necroptotic stimulus (TSI) for $3 \mathrm{hrs}$, followed by UBA-pulldown and USP21 digestion. Antibody (D6E3G, Cell Signaling Technology) was used here to detect MLKL phosphorylation. Representative of 2 independent experiments.

D $M L K L^{-/}$HT29 cells were stably transfected with indicated doxycycline inducible $M L K L$ alleles (here phosphor-mimic human MLKL mutant T357E/S358E indicated as MLKL ${ }^{\text {TSEE }}$ ) and treated with doxycycline $(100 \mathrm{ng} / \mathrm{mL}) \pm$ TSI (added simultaneously). Representative of 3 independent experiments.

\section{References}

Bertrand MJ, Milutinovic S, Dickson KM, Ho WC, Boudreault A, Durkin J, Gillard JW, Jaquith JB, Morris SJ, Barker PA (2008) ClAP1 and CIAP2 facilitate cancer cell survival by functioning

34 as E3 ligases that promote RIP1 ubiquitination. Mol Cell 30: 689-700 
1 Cai Z, Jitkaew S, Zhao J, Chiang HC, Choksi S, Liu J, Ward Y, Wu LG, Liu ZG (2014) Plasma

2 membrane translocation of trimerized MLKL protein is required for TNF-induced necroptosis.

3 Nat Cell Biol 16: 55-65

4 Chen X, Li W, Ren J, Huang D, He WT, Song Y, Yang C, Li W, Zheng X, Chen P et al (2014)

5 Translocation of mixed lineage kinase domain-like protein to plasma membrane leads to

6 necrotic cell death. Cell research 24: 105-121

7 Cox J, Neuhauser N, Michalski A, Scheltema RA, Olsen JV, Mann M (2011) Andromeda: a

8 peptide search engine integrated into the MaxQuant environment. J Proteome Res 10:

9 1794-1805

10 Davies KA, Fitzgibbon C, Young SN, Garnish SE, Yeung W, Coursier D, Birkinshaw RW,

Sandow JJ, Lehmann WIL, Liang LY et al (2020) Distinct pseudokinase domain conformations underlie divergent activation mechanisms among vertebrate MLKL orthologues. Nat Commun 11: 3060

Davies KA, Tanzer MC, Griffin MDW, Mok YF, Young SN, Qin R, Petrie EJ, Czabotar PE, Silke J, Murphy JM (2018) The brace helices of MLKL mediate interdomain communication and oligomerisation to regulate cell death by necroptosis. Cell Death Differ 25: 1567-1580 Delconte RB, Kolesnik TB, Dagley LF, Rautela J, Shi W, Putz EM, Stannard K, Zhang JG, Teh C, Firth $\mathrm{M}$ et al (2016) CIS is a potent checkpoint in NK cell-mediated tumor immunity. Nat Immunol 17: 816-824

Dondelinger Y, Darding M, Bertrand MJ, Walczak H (2016) Poly-ubiquitination in TNFR1mediated necroptosis. Cell Mol Life Sci 73: 2165-2176

Dovey CM, Diep J, Clarke BP, Hale AT, McNamara DE, Guo H, Brown NW, Jr., Cao JY, Grace CR, Gough PJ et al (2018) MLKL Requires the Inositol Phosphate Code to Execute Necroptosis. Mol Cell 70: 936-948 e937

Draber P, Kupka S, Reichert M, Draberova H, Lafont E, de Miguel D, Spilgies L, Surinova S, Taraborrelli L, Hartwig Tet al (2015) LUBAC-Recruited CYLD and A20 Regulate Gene Activation and Cell Death by Exerting Opposing Effects on Linear Ubiquitin in Signaling Complexes. Cell Rep 13: 2258-2272

Fan W, Guo J, Gao B, Zhang W, Ling L, Xu T, Pan C, Li L, Chen S, Wang H et al (2019) Flotillinmediated endocytosis and ALIX-syntenin-1-mediated exocytosis protect the cell membrane from damage caused by necroptosis. Science signaling 12

Gong YN, Guy C, Olauson H, Becker JU, Yang M, Fitzgerald P, Linkermann A, Green DR (2017) ESCRT-III Acts Downstream of MLKL to Regulate Necroptotic Cell Death and Its Consequences. Cell 169: 286-300 e216

Haas TL, Emmerich CH, Gerlach B, Schmukle AC, Cordier SM, Rieser E, Feltham R, Vince J, Warnken $U$, Wenger T et al (2009) Recruitment of the linear ubiquitin chain assembly complex stabilizes the TNF-R1 signaling complex and is required for TNF-mediated gene induction. Mol Cell 36: 831-844

Haglund K, Sigismund S, Polo S, Szymkiewicz I, Di Fiore PP, Dikic I (2003) Multiple monoubiquitination of RTKs is sufficient for their endocytosis and degradation. Nat Cell Biol 5: 461-466

Hancock JF, Cadwallader K, Paterson H, Marshall CJ (1991) A CAAX or a CAAL motif and a second signal are sufficient for plasma membrane targeting of ras proteins. EMBO $\mathrm{J} 10$ : 4033-4039

45 Hildebrand JM, Kauppi M, Majewski IJ, Liu Z, Cox AJ, Miyake S, Petrie EJ, Silk MA, Li Z, Tanzer

$46 \mathrm{MC}$ et al (2020) A missense mutation in the MLKL brace region promotes lethal neonatal

47 inflammation and hematopoietic dysfunction. Nat Commun 11: 3150 
1 Hildebrand JM, Tanzer MC, Lucet IS, Young SN, Spall SK, Sharma P, Pierotti C, Garnier JM,

2 Dobson RC, Webb Al et al (2014) Activation of the pseudokinase MLKL unleashes the four-

3 helix bundle domain to induce membrane localization and necroptotic cell death. Proc Natl

4 Acad SciU S A 111: 15072-15077

5 Hjerpe R, Aillet F, Lopitz-Otsoa F, Lang V, England P, Rodriguez MS (2009) Efficient

6 protection and isolation of ubiquitylated proteins using tandem ubiquitin-binding entities.

7 EMBO Rep 10: 1250-1258

8 Holler N, Zaru R, Micheau O, Thome M, Attinger A, Valitutti S, Bodmer JL, Schneider P, Seed

9 B, Tschopp J (2000) Fas triggers an alternative, caspase-8-independent cell death pathway

10 using the kinase RIP as effector molecule. Nat Immunol 1: 489-495

11 Hospenthal MK, Mevissen TET, Komander D (2015) Deubiquitinase-based analysis of

12 ubiquitin chain architecture using Ubiquitin Chain Restriction (UbiCRest). Nat Protoc 10:

13 349-361

Lysine 63-linked polyubiquitination is required for EGF receptor degradation. Proc Natl Acad Sci U S A 110: 15722-15727

Ikeda F, Deribe YL, Skanland SS, Stieglitz B, Grabbe C, Franz-Wachtel M, van Wijk SJ, Goswami P, Nagy V, Terzic J et al (2011) SHARPIN forms a linear ubiquitin ligase complex regulating NF-kappaB activity and apoptosis. Nature 471: 637-641

Jacobsen AV, Lowes KN, Tanzer MC, Lucet IS, Hildebrand JM, Petrie EJ, van Delft MF, Liu Z, Conos SA, Zhang JG et al (2016) HSP90 activity is required for MLKL oligomerisation and membrane translocation and the induction of necroptotic cell death. Cell Death Dis 7: e2051 Kaczmarek A, Vandenabeele P, Krysko DV (2013) Necroptosis: the release of damageassociated molecular patterns and its physiological relevance. Immunity 38: 209-223 Kaiser WJ, Sridharan H, Huang C, Mandal P, Upton JW, Gough PJ, Sehon CA, Marquis RW, Bertin J, Mocarski ES (2013) Toll-like receptor 3-mediated necrosis via TRIF, RIP3, and MLKL. J Biol Chem 288: 31268-31279

Kedzierski L, Tate MD, Hsu AC, Kolesnik TB, Linossi EM, Dagley L, Dong Z, Freeman S, Infusini G, Starkey MR et al (2017) Suppressor of Cytokine Signaling (SOCS)5 ameliorates influenza infection via inhibition of EGFR signaling. Elife 6

Keusekotten K, Elliott PR, Glockner L, Fiil BK, Damgaard RB, Kulathu Y, Wauer T, Hospenthal MK, Gyrd-Hansen M, Krappmann D et al (2013) OTULIN antagonizes LUBAC signaling by specifically hydrolyzing Met1-linked polyubiquitin. Cell 153: 1312-1326

Lacey D, Hickey P, Arhatari BD, O'Reilly LA, Rohrbeck L, Kiriazis H, Du XJ, Bouillet P (2015)

Spontaneous retrotransposon insertion into TNF 3'UTR causes heart valve disease and chronic polyarthritis. Proc Natl Acad Sci U S A 112: 9698-9703

Lawlor KE, Khan N, Mildenhall A, Gerlic M, Croker BA, D'Cruz AA, Hall C, Kaur Spall S, Anderton H, Masters SL et al (2015) RIPK3 promotes cell death and NLRP3 inflammasome activation in the absence of MLKL. Nat Commun 6: 6282

Li D, Xu T, Cao Y, Wang H, Li L, Chen S, Wang X, Shen Z (2015) A cytosolic heat shock protein 90 and cochaperone CDC37 complex is required for RIP3 activation during necroptosis. Proc Natl Acad Sci U S A 112: 5017-5022

Liu S, Liu H, Johnston A, Hanna-Addams S, Reynoso E, Xiang Y, Wang Z (2017) MLKL forms disulfide bond-dependent amyloid-like polymers to induce necroptosis. Proc Natl Acad SciU S A 114: E7450-E7459

46 Liu Z, Silke J, Hildebrand JM (2018) Methods for Studying TNF-Mediated Necroptosis in

47 Cultured Cells. Methods Mol Biol 1857: 53-61 
1 Menon MB, Gaestel M (2018) MK2-TNF-Signaling Comes Full Circle. Trends Biochem Sci 43:

$2 \quad 170-179$

3 Morrow ME, Morgan MT, Clerici M, Growkova K, Yan M, Komander D, Sixma TK, Simicek M,

$4 \quad$ Wolberger $\mathrm{C}$ (2018) Active site alanine mutations convert deubiquitinases into high-affinity

5 ubiquitin-binding proteins. EMBO Rep 19

6 Mosesson $Y$, Yarden $Y$ (2006) Monoubiquitylation: a recurrent theme in membrane protein

7 transport. Isr Med Assoc J 8: 233-237

8 Moujalled DM, Cook WD, Murphy JM, Vaux DL (2014) Necroptosis induced by RIPK3

9 requires MLKL but not Drp1. Cell Death Dis 5: e1086

10 Murai S, Yamaguchi Y, Shirasaki Y, Yamagishi M, Shindo R, Hildebrand JM, Miura R, Nakabayashi O, Totsuka M, Tomida T et al (2018) A FRET biosensor for necroptosis uncovers two different modes of the release of DAMPs. Nat Commun 9: 4457 Murphy JM, Czabotar PE, Hildebrand JM, Lucet IS, Zhang JG, Alvarez-Diaz S, Lewis R, Lalaoui $\mathrm{N}$, Metcalf D, Webb Al et al (2013) The pseudokinase MLKL mediates necroptosis via a molecular switch mechanism. Immunity 39: 443-453

Oh E, Akopian D, Rape M (2018) Principles of Ubiquitin-Dependent Signaling. Annu Rev Cell Dev Biol 34: 137-162

Onizawa M, Oshima S, Schulze-Topphoff U, Oses-Prieto JA, Lu T, Tavares R, Prodhomme T, Duong B, Whang MI, Advincula R et al (2015) The ubiquitin-modifying enzyme A20 restricts ubiquitination of the kinase RIPK3 and protects cells from necroptosis. Nat Immunol 16: 618-627

Orozco S, Yatim N, Werner MR, Tran H, Gunja SY, Tait SW, Albert ML, Green DR, Oberst A (2014) RIPK1 both positively and negatively regulates RIPK3 oligomerization and necroptosis. Cell Death Differ 21: 1511-1521

Perez-Riverol Y, Csordas A, Bai J, Bernal-Llinares M, Hewapathirana S, Kundu DJ, Inuganti A, Griss J, Mayer G, Eisenacher M et al (2019) The PRIDE database and related tools and resources in 2019: improving support for quantification data. Nucleic Acids Res 47: D442D450

Petersen SL, Chen TT, Lawrence DA, Marsters SA, Gonzalvez F, Ashkenazi A (2015) TRAF2 is a biologically important necroptosis suppressor. Cell Death Differ 22: 1846-1857

Petrie EJ, Birkinshaw RW, Koide A, Denbaum E, Hildebrand JM, Garnish SE, Davies KA, Sandow JJ, Samson AL, Gavin X et al (2020) Identification of MLKL membrane translocation as a checkpoint in necroptotic cell death using Monobodies. Proc Natl Acad Sci U S A 117: 8468-8475

Petrie EJ, Czabotar PE, Murphy JM (2019a) The Structural Basis of Necroptotic Cell Death Signaling. Trends Biochem Sci 44: 53-63

Petrie EJ, Sandow JJ, Jacobsen AV, Smith BJ, Griffin MDW, Lucet IS, Dai W, Young SN, Tanzer MC, Wardak A et al (2018) Conformational switching of the pseudokinase domain promotes human MLKL tetramerization and cell death by necroptosis. Nat Commun 9: 2422

Petrie EJ, Sandow JJ, Lehmann WIL, Liang LY, Coursier D, Young SN, Kersten WJA, Fitzgibbon C, Samson AL, Jacobsen AV et al (2019b) Viral MLKL Homologs Subvert Necroptotic Cell Death by Sequestering Cellular RIPK3. Cell Rep 28: 3309-3319 e3305

42 Petroski MD, Deshaies RJ (2003) Context of multiubiquitin chain attachment influences the rate of Sic1 degradation. Mol Cell 11: 1435-1444

Samson AL, Zhang Y, Geoghegan ND, Gavin XJ, Davies KA, Mlodzianoski MJ, Whitehead LW, Frank D, Garnish SE, Fitzgibbon C et al (2020) MLKL trafficking and accumulation at the plasma membrane control the kinetics and threshold for necroptosis. Nat Commun 11: 3151 
1 Sapmaz A, Berlin I, Bos E, Wijdeven RH, Janssen H, Konietzny R, Akkermans JJ, Erson-Bensan

2 AE, Koning RI, Kessler BM et al (2019) USP32 regulates late endosomal transport and

3 recycling through deubiquitylation of Rab7. Nat Commun 10: 1454

4 Schagger $\mathrm{H}$, von Jagow $\mathrm{G}$ (1991) Blue native electrophoresis for isolation of membrane

5 protein complexes in enzymatically active form. Anal Biochem 199: 223-231

6 Segawa K, Nagata S (2015) An Apoptotic 'Eat Me' Signal: Phosphatidylserine Exposure.

7 Trends Cell Biol 25: 639-650

8 Stafford CA, Lawlor KE, Heim VJ, Bankovacki A, Bernardini JP, Silke J, Nachbur U (2018) IAPS

9 Regulate Distinct Innate Immune Pathways to Co-ordinate the Response to Bacterial

10 Peptidoglycans. Cell Rep 22: 1496-1508

11 Sun X, Yin J, Starovasnik MA, Fairbrother WJ, Dixit VM (2002) Identification of a novel homotypic interaction motif required for the phosphorylation of receptor-interacting protein (RIP) by RIP3. J Biol Chem 277: 9505-9511

Swatek KN, Komander D (2016) Ubiquitin modifications. Cell Res 26: 399-422

Tanzer MC, Matti I, Hildebrand JM, Young SN, Wardak A, Tripaydonis A, Petrie EJ, Mildenhall AL, Vaux DL, Vince JE et al (2016) Evolutionary divergence of the necroptosis effector MLKL. Cell Death Differ 23: 1185-1197

Tanzer MC, Tripaydonis A, Webb Al, Young SN, Varghese LN, Hall C, Alexander WS, Hildebrand JM, Silke J, Murphy JM (2015) Necroptosis signalling is tuned by phosphorylation of MLKL residues outside the pseudokinase domain activation loop. Biochem J 471: 255-265 Tokunaga F, Sakata S, Saeki Y, Satomi Y, Kirisako T, Kamei K, Nakagawa T, Kato M, Murata S, Yamaoka S et al (2009) Involvement of linear polyubiquitylation of NEMO in NF-kappaB activation. Nat Cell Biol 11: 123-132

Vanden Berghe T, Kaiser WJ, Bertrand MJ, Vandenabeele P (2015) Molecular crosstalk between apoptosis, necroptosis, and survival signaling. Mol Cell Oncol 2: e975093 Vandenabeele P, Riquet F, Cappe B (2017) Necroptosis: (Last) Message in a Bubble. Immunity 47: 1-3

Wang H, Sun L, Su L, Rizo J, Liu L, Wang LF, Wang FS, Wang X (2014) Mixed lineage kinase domain-like protein MLKL causes necrotic membrane disruption upon phosphorylation by RIP3. Mol Cell 54: 133-146

Wang Z, Jiang H, Chen S, Du F, Wang X (2012) The mitochondrial phosphatase PGAM5 functions at the convergence point of multiple necrotic death pathways. Cell 148: 228-243 Weber K, Roelandt R, Bruggeman I, Estornes Y, Vandenabeele P (2018) Nuclear RIPK3 and MLKL contribute to cytosolic necrosome formation and necroptosis. Commun Biol 1: 6 Wilkinson KD, Tashayev VL, O'Connor LB, Larsen CN, Kasperek E, Pickart CM (1995) Metabolism of the polyubiquitin degradation signal: structure, mechanism, and role of isopeptidase T. Biochemistry 34: 14535-14546

Wisniewski JR, Zougman A, Nagaraj N, Mann M (2009) Universal sample preparation method for proteome analysis. Nat Methods 6: 359-362

Wright LP, Philips MR (2006) Thematic review series: lipid posttranslational modifications. CAAX modification and membrane targeting of Ras. J Lipid Res 47: 883-891

Wu G, Xu G, Schulman BA, Jeffrey PD, Harper JW, Pavletich NP (2003) Structure of a betaTrCP1-Skp1-beta-catenin complex: destruction motif binding and lysine specificity of the SCF(beta-TrCP1) ubiquitin ligase. Mol Cell 11: 1445-1456

Wu XN, Yang ZH, Wang XK, Zhang Y, Wan H, Song Y, Chen X, Shao J, Han J (2014) Distinct roles of RIP1-RIP3 hetero- and RIP3-RIP3 homo-interaction in mediating necroptosis. Cell

47 Death Differ 21: 1709-1720 
1 Ye Y, Akutsu M, Reyes-Turcu F, Enchev RI, Wilkinson KD, Komander D (2011) Polyubiquitin

2 binding and cross-reactivity in the USP domain deubiquitinase USP21. EMBO Rep 12: 350-

\section{$3 \quad 357$}

4 Ye Y, Blaser G, Horrocks MH, Ruedas-Rama MJ, Ibrahim S, Zhukov AA, Orte A, Klenerman D, 5 Jackson SE, Komander D (2012) Ubiquitin chain conformation regulates recognition and 6 activity of interacting proteins. Nature 492: 266-270

7 Yoon S, Bogdanov K, Kovalenko A, Wallach D (2016) Necroptosis is preceded by nuclear

8 translocation of the signaling proteins that induce it. Cell Death Differ 23: 253-260

9 Yoon S, Kovalenko A, Bogdanov K, Wallach D (2017) MLKL, the Protein that Mediates

10 Necroptosis, Also Regulates Endosomal Trafficking and Extracellular Vesicle Generation.

11 Immunity 47: 51-65 e57

12 Zargarian S, Shlomovitz I, Erlich Z, Hourizadeh A, Ofir-Birin Y, Croker BA, Regev-Rudzki N, 13 Edry-Botzer L, Gerlic M (2017) Phosphatidylserine externalization, "necroptotic bodies"

14 release, and phagocytosis during necroptosis. PLoS Biol 15: e2002711 
Figure 1. MLKL undergoes ubiquitylation during necroptosis

bioRxiv preprint doi: https://doi.org/10.1101/2021.05.01.442209; this version posted May 1, 2021. The copyright holder for this preprint (which was not certified by peer review) is the author/funder. All rights reserved. No reuse allowed without permission.

\section{A WT MDF}

\section{TNF}

Compound $\mathrm{A}-\mathrm{C}_{-}+\mathrm{C}_{-}+$

IDN-6556 - $\quad-\quad+\quad+\quad++$

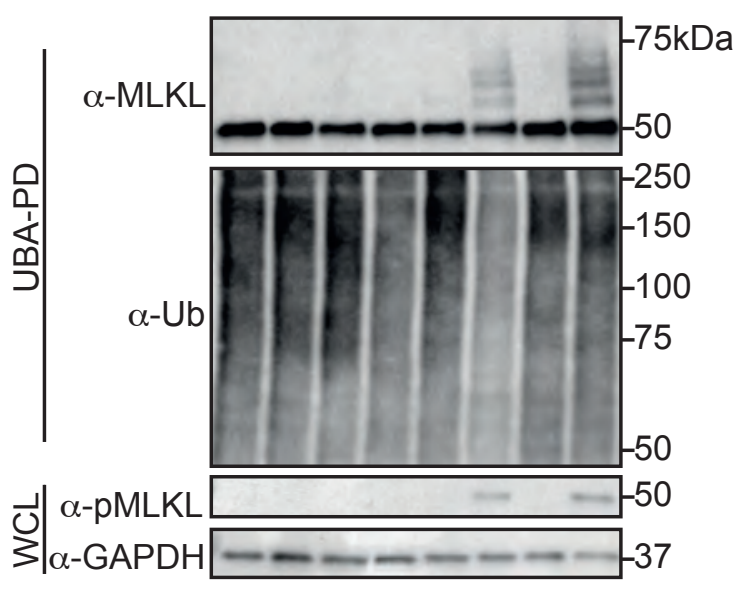

C

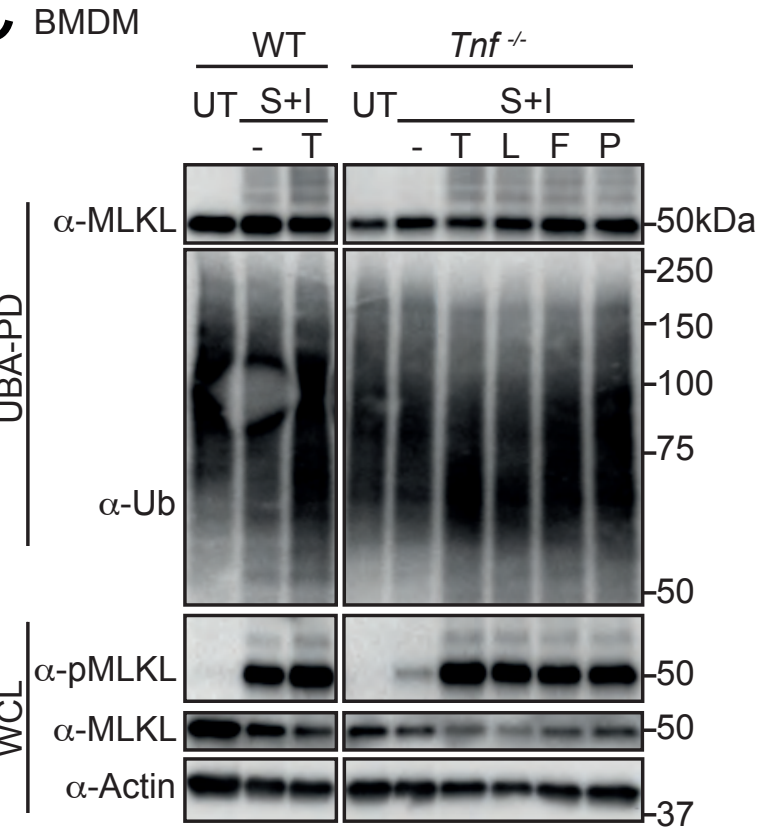

B MDF
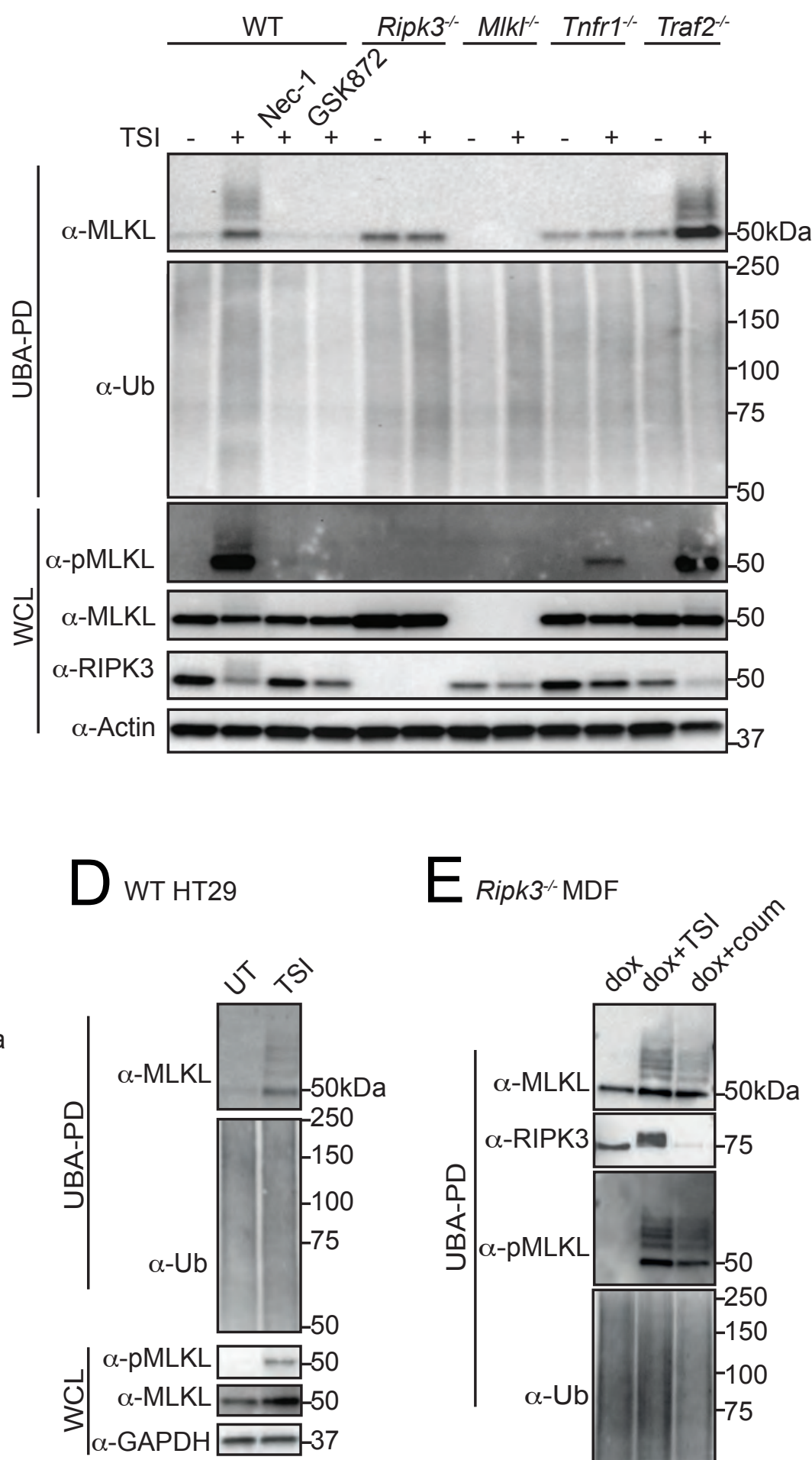

E Ripk3- MDF

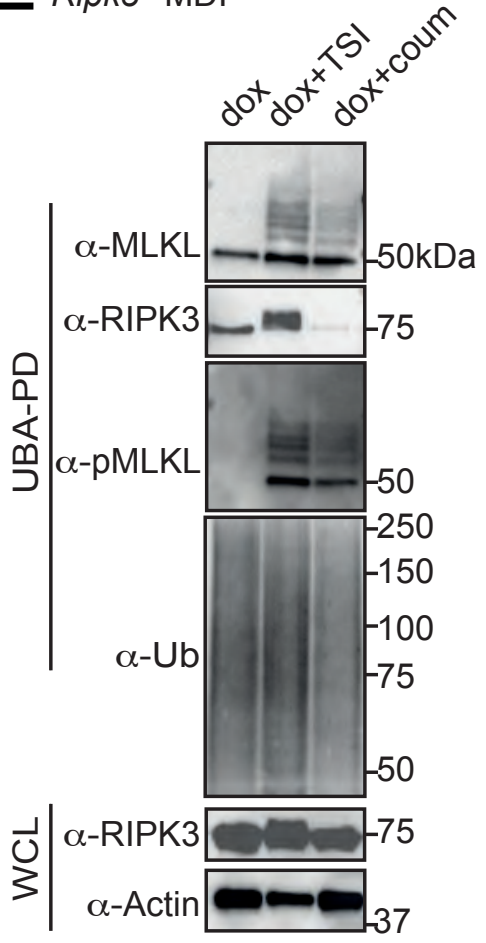




\section{Supplementary Figure 1. Cell death time course of MDFs and HT29 cells following necroptotic stimulation}

bioRxiv preprint doi: https://doi.org/10.1101/2021.05.01.442209; this version posted May 1, 2021. The copyright holder for this preprint (which was not certified by peer review) is the author/funder. All rights reserved. No reuse allowed without permission.

A WT MDF

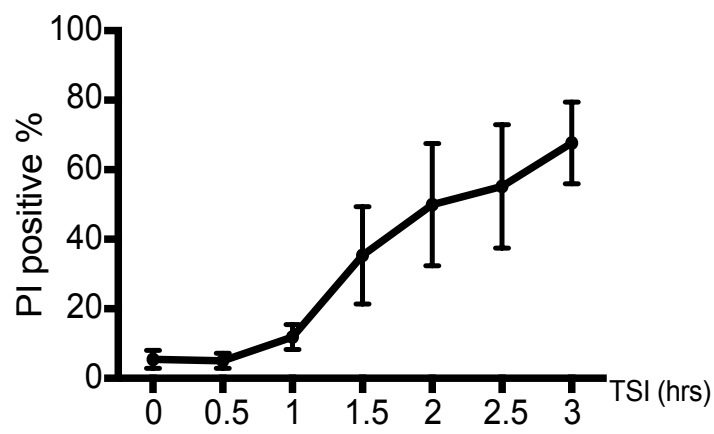

B WT HT29

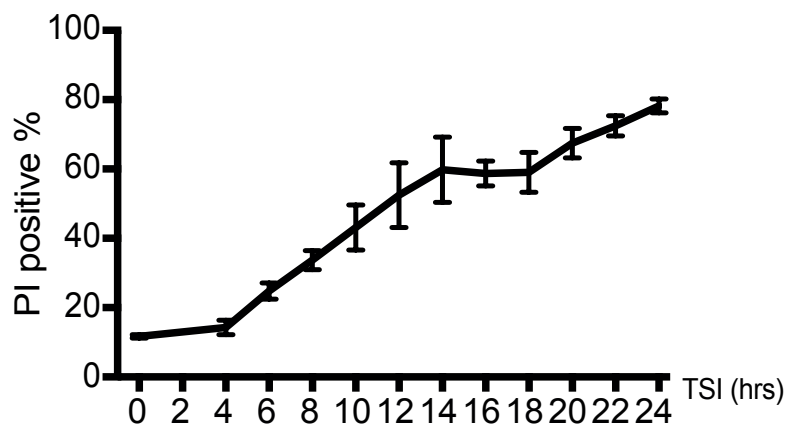


Figure 2. MLKL is mono-ubiquitylated at multiple sites

bioRxiv preprint doi: https://doi.org/10.1101/2021.05.01.442209; this version posted May 1, 2021. The copyright holder for this preprint (which was not certified by peer review) is the author/funder. All rights reserved. No reuse allowed without permission.

\begin{tabular}{|c|c|}
\hline DUBs & Substrate linkage type \\
\hline USP21 & All ubiquitin linkages \\
\hline vOTU & All poly-ubiquitylation except M1 linkage \\
\hline OTULIN & M1 \\
\hline OTUD1 & K63 \\
\hline OTUD2 & K27 (K11, K29, K33) \\
\hline OTUD3 & K6 (K11) \\
\hline OTUB1 & K48 \\
\hline TRABID & K29, K33 (K69) \\
\hline Cezanne & K11 \\
\hline
\end{tabular}
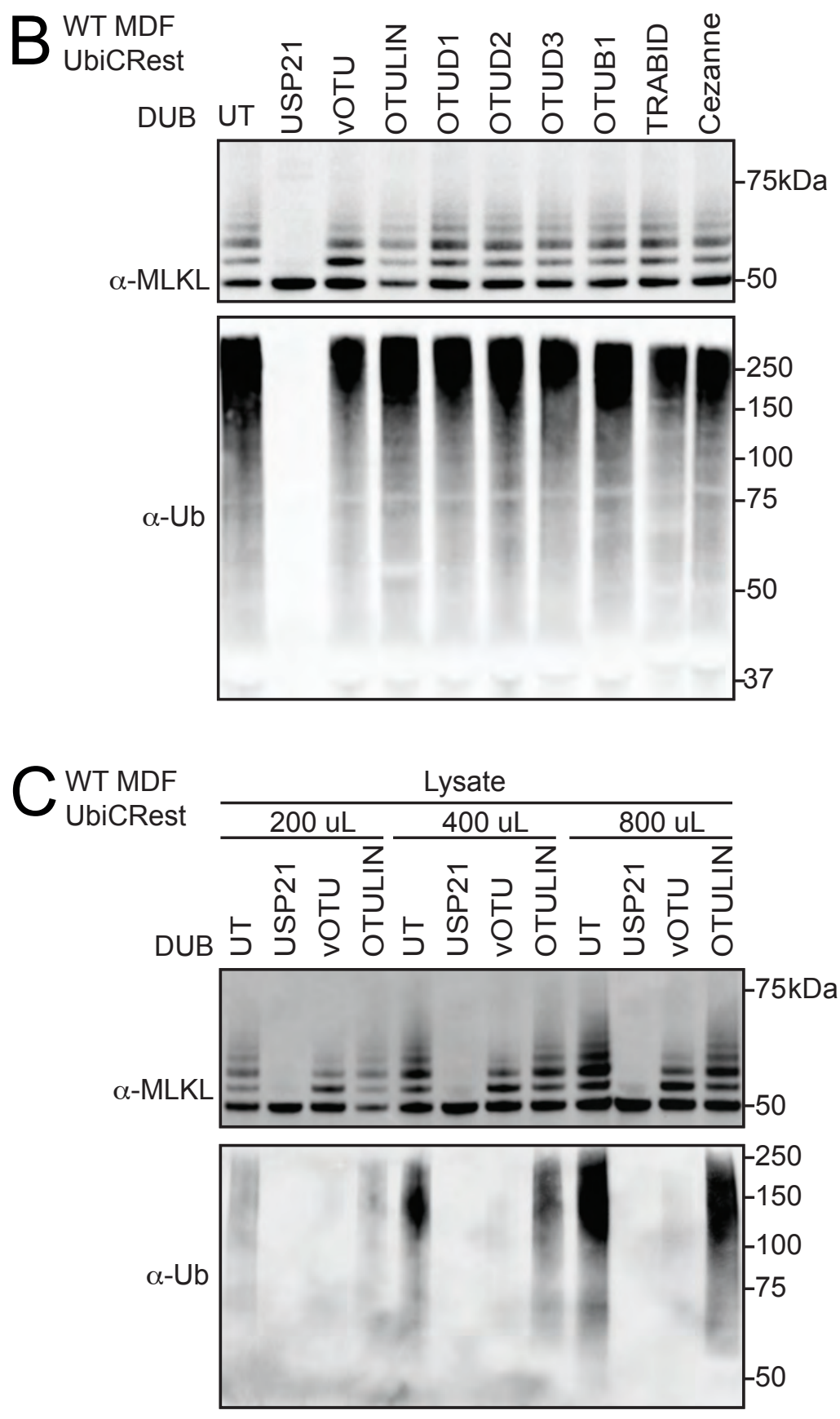
Figure 3. MLKL ubiquitylation accumulates in crude membranes and can be digested by USP21 located on biological membranes.

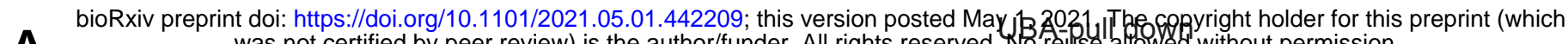

$\mathrm{A}_{\mathrm{WT} M \mathrm{MP}}$ was not certified by peer review) is the author/funder. All rights reserved. No relise allowed without permission.

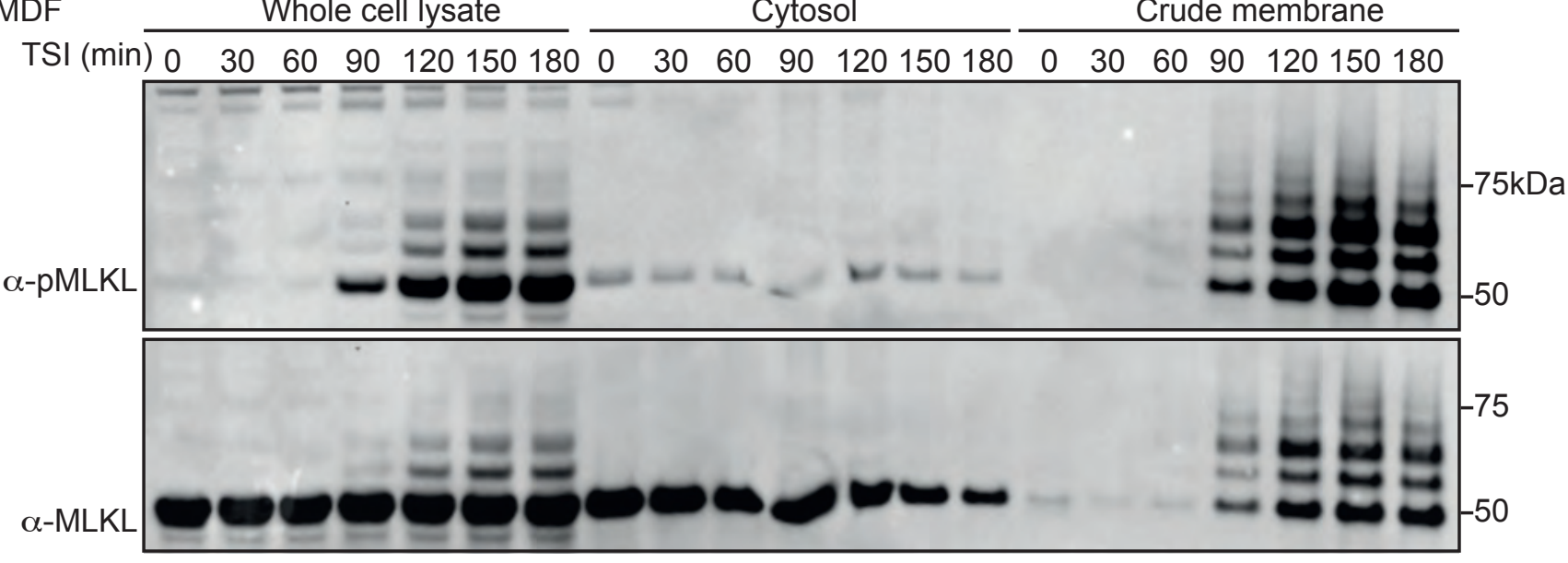

Whole cell lysate

Cytosol input

Crude membrane input

TSI (min) $0 \begin{array}{lllllllllllllllll} & 0 & 60 & 90 & 120150180 & 0 & 30 & 60 & 90 & 120 & 150180 & 0 & 30 & 60 & 90 & 120 & 150180\end{array}$
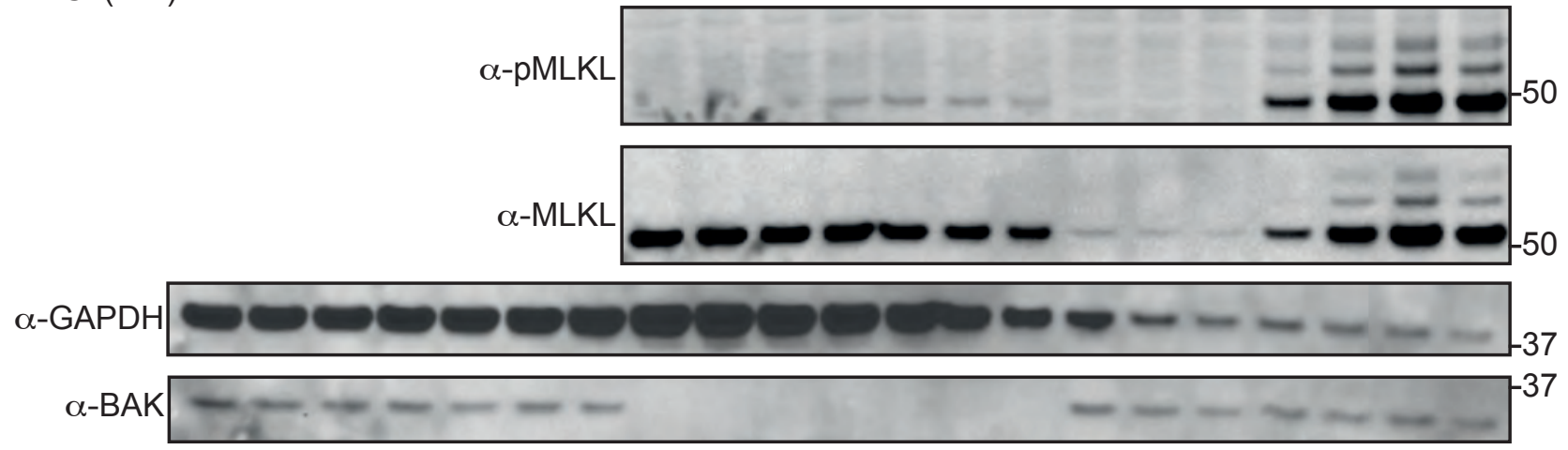

$B_{\text {WTMPF }}$

$\frac{\mathrm{C}}{\frac{\mathrm{UT}}{\mathrm{WT}} \frac{\mathrm{CR}}{\frac{\mathrm{TSI}}{\mathrm{WT}}} \frac{\mathrm{CR}}{\frac{\mathrm{UT}}{\mathrm{WT}} \frac{\mathrm{CR}}{\frac{\mathrm{C}}{-+}} \frac{\mathrm{TSI}}{-+\frac{\mathrm{CR}}{-+}}}}$ USP21-CaaX

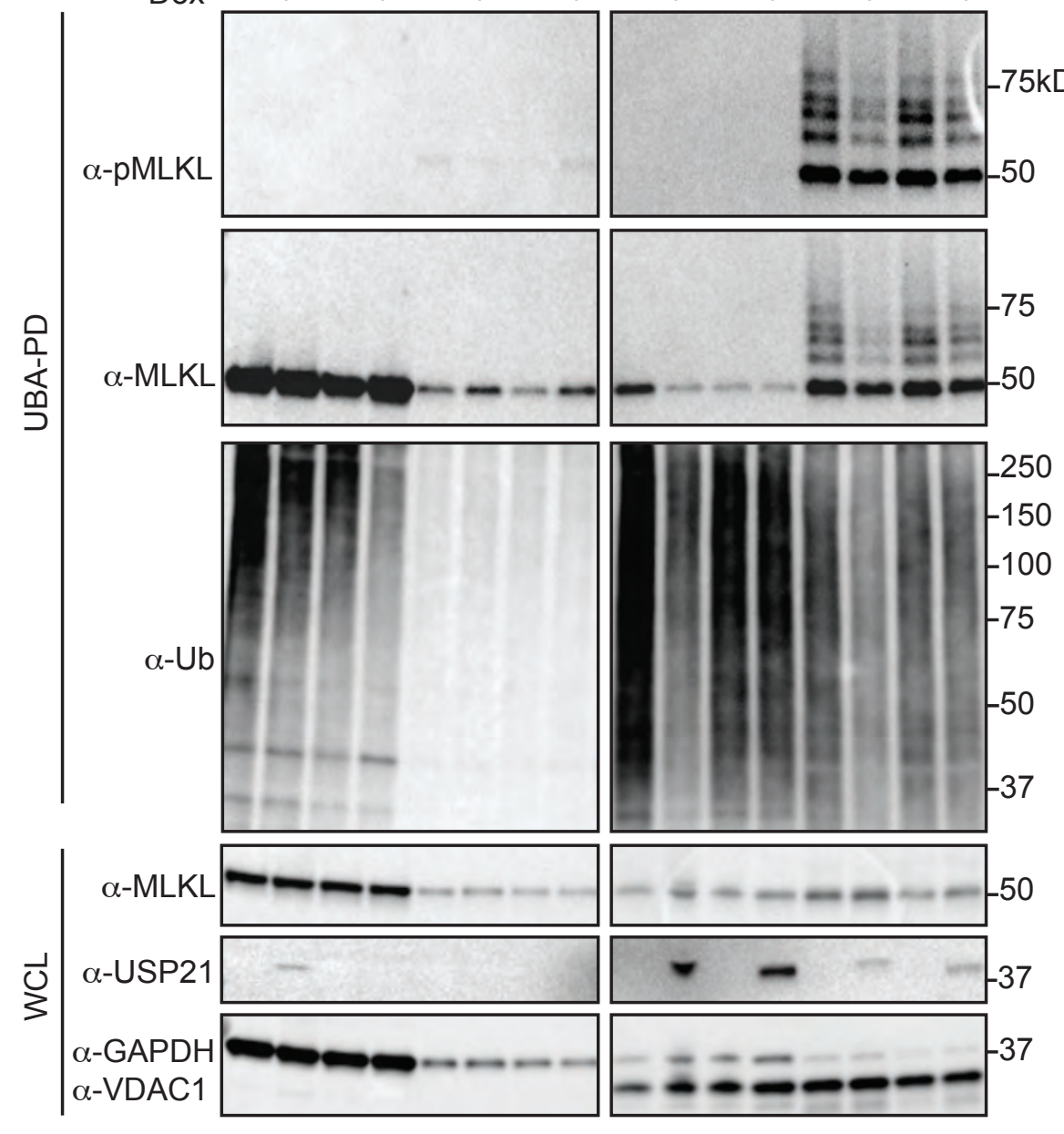


Supplementary Figure 3. USP21-CaaX expression does not alter the kinetics of TNF induced apoptosis or necroptosis in MDFs

bioRxiv preprint doi: https://doi.org/10.1101/2021.05.01.442209; this version posted May 1, 2021. The copyright holder for this preprint (which was not certified by peer review) is the author/funder. All rights reserved. No reuse allowed without permissign $\mathrm{T}$ MF

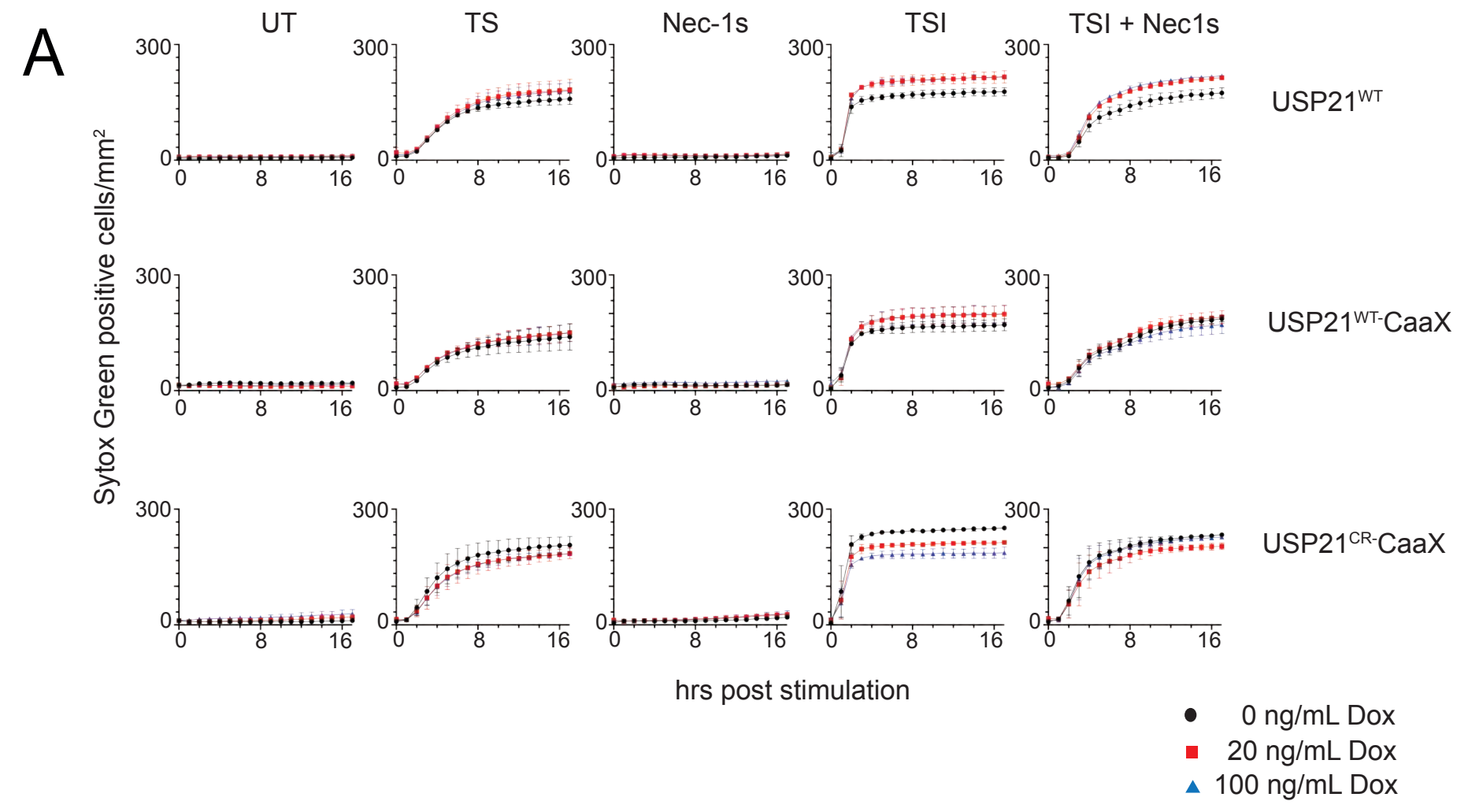


Figure 4. MLKL oligomerization drives its necroptosis specific ubiquitylation

bioRxiv preprint doi: https://doi.org/10.1101/2021.05.01.442209; this version posted May 1, 2021. The copyright holder for this preprint (which was not certified by peer review) is the author/funder. All rights reserved. No reuse allowed withp 4 bgekmission.

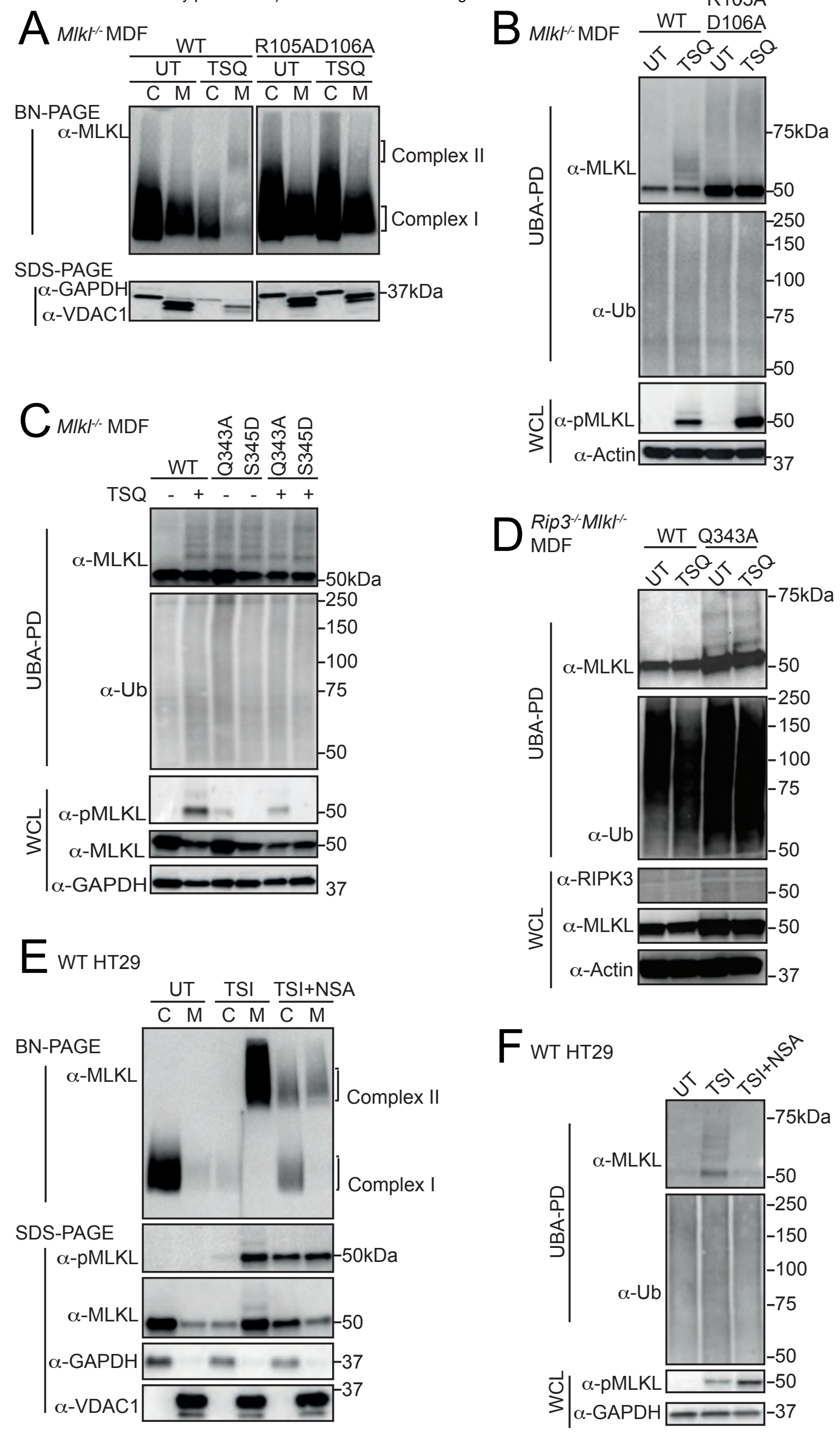


Supplementary Figure 4. MLKL oligomerization drives its necroptosis specific ubiquitylation

bioRxiv preprint doi: https://doi.org/10.1101/2021.05.01.442209; this version posted May 1, 2021. The copyright holder for this preprint (which was not certified by peer review) is the author/funder. All rights reserved. No reuse allowed without permission.
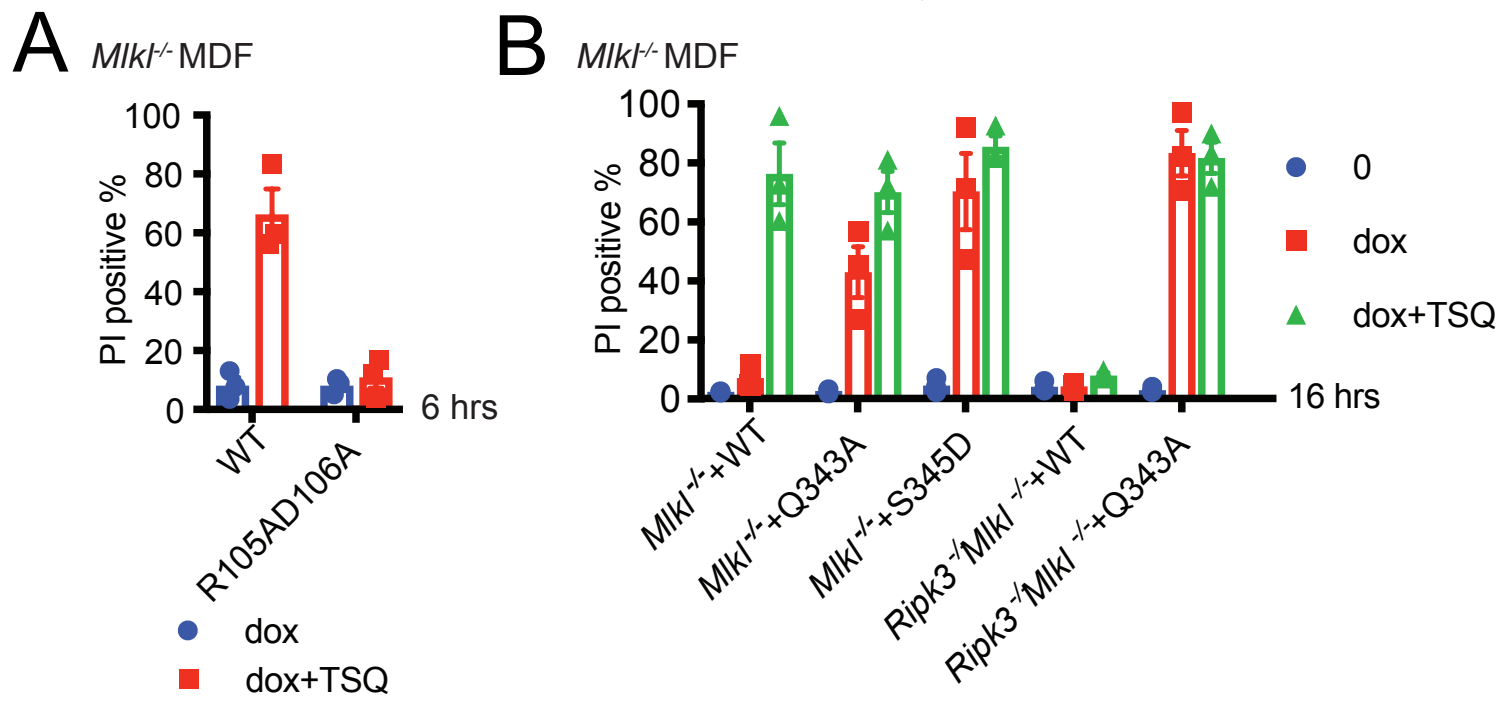
Figure 5. MLKL ubiquitylation does not lead to cell death, but correlates with the turnover of activated MLKL

bioRxiv preprint doi: https://doi.org/10.1101/2021.05.01.442209; this version posted May 1, 2021. The copyright holder for this preprint (which was not certified by peer review) is the author/funder. All rights reserved. No reuse allowed without permission. E109A
A Mkk'MDF
B
MIkr-MDF

C MIk $r^{-\mu M D F}$
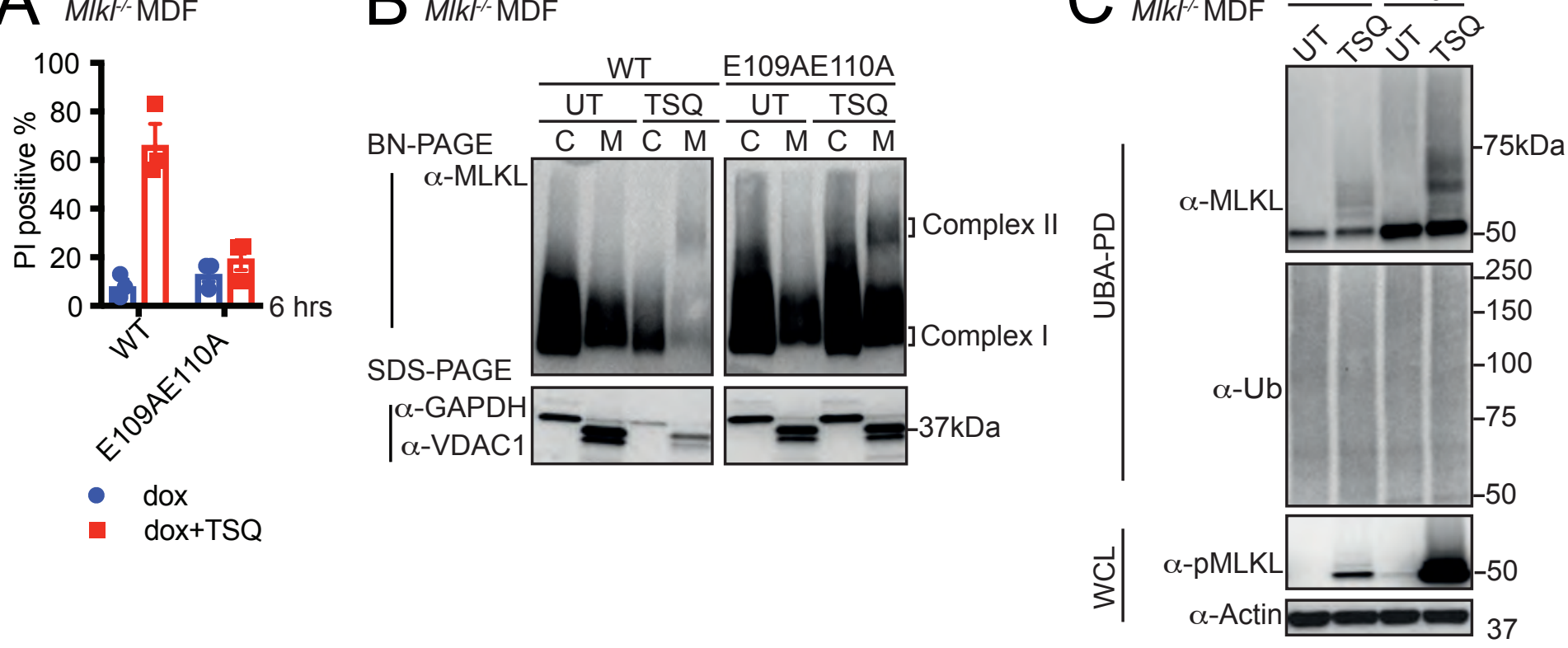

D

MIk ${ }^{-\sim M D F+}$

N-FLAG MLKL

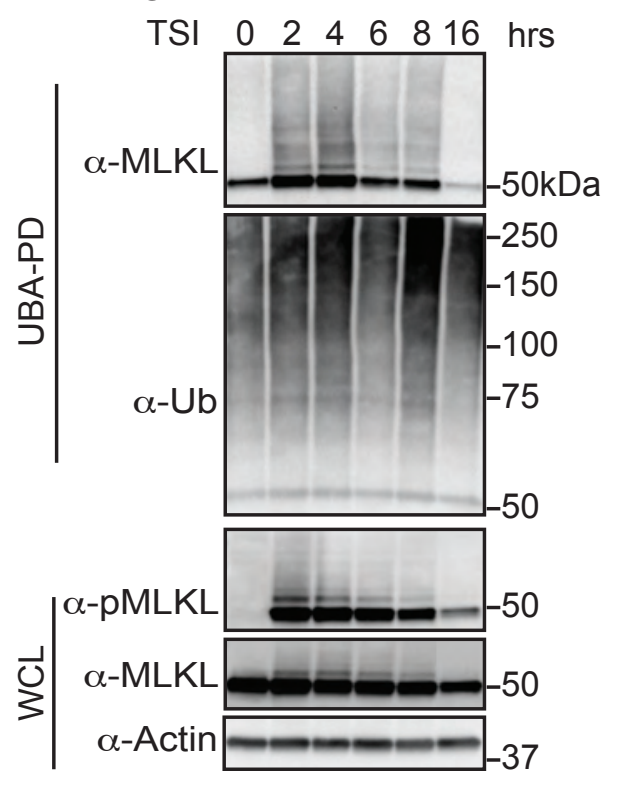

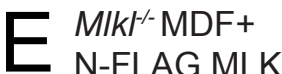

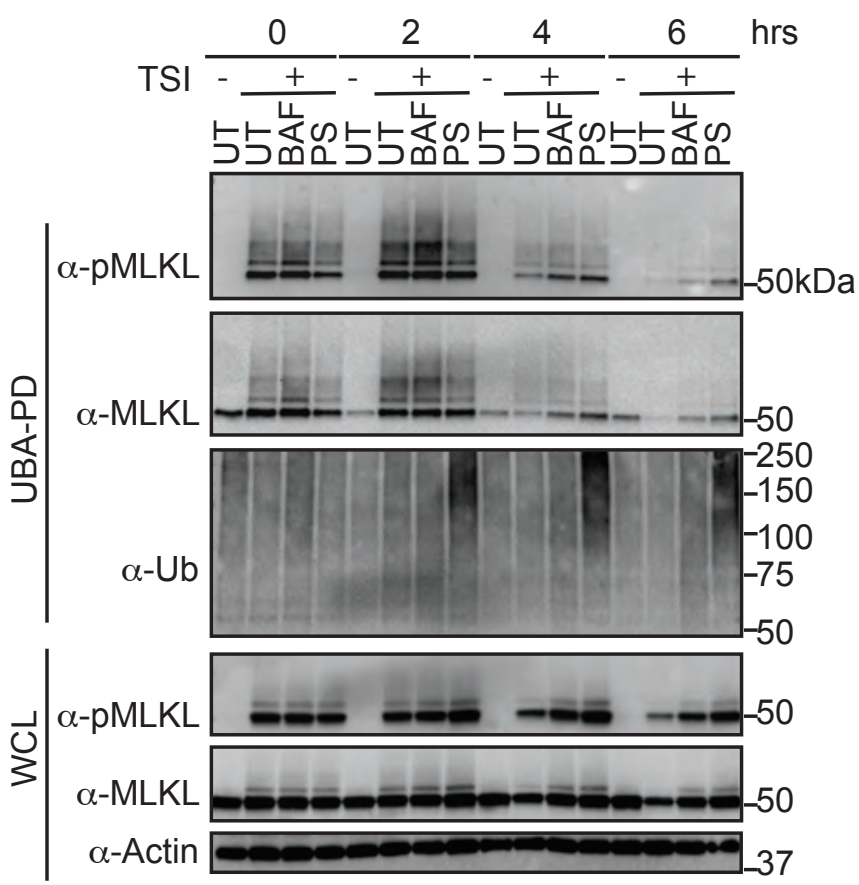


Supplementary Figure 5. N-FLAG MLKL behaves like WT MLKL but does not induce cell death following necroptotic stimulation

bioRxiv preprint doi: https://doi.org/10.1101/2021.05.01.442209; this version posted May 1, 2021. The copyright holder for this preprint (which was not certified by peer review) is the author/funder. All rights reserved. No reuse allowed without permission.

A

MIkl-MDF

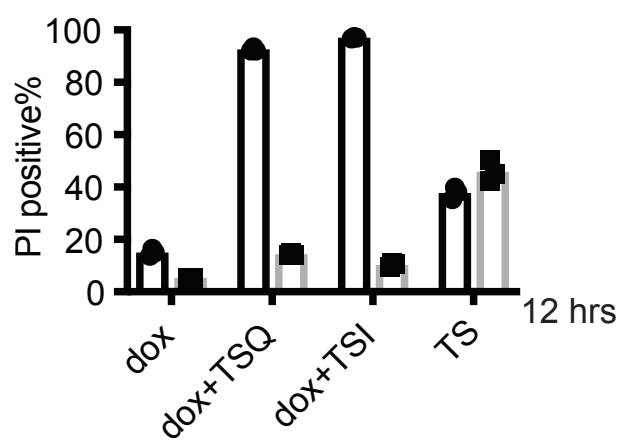

- WT MLKL

- N-FLAG MLKL
$B$ Mlkt-MDF WT N-FLAG

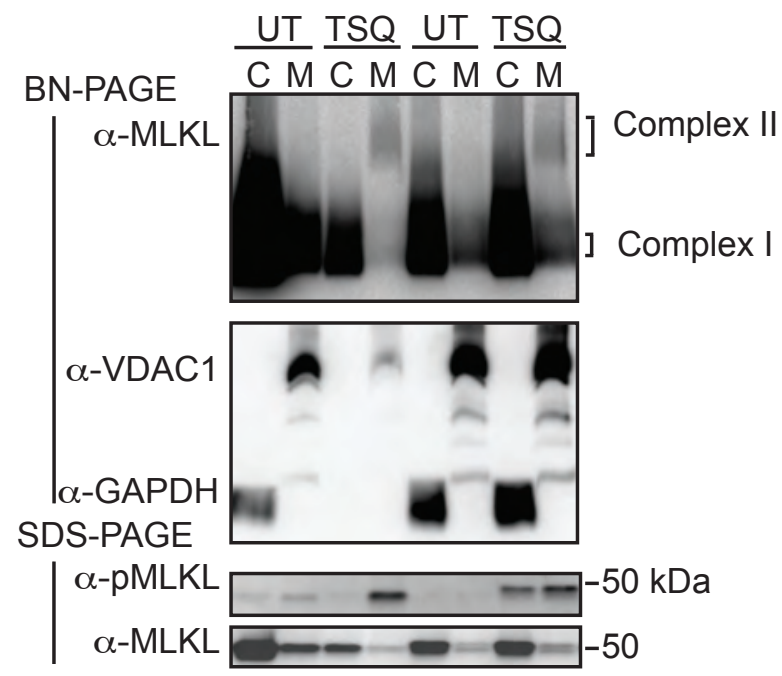


Figure 6. Simultaneous arginine replacement of 4 ubiquitylation sites on the mMLKL 4HB domain does not prevent necroptosis-induced ubiquitylation

bioRxiv preprint doi: https://doi.org/10.1101/2021.05.01.442209; this version posted May 1, 2021. The copyright holder for this preprint (which

A was not certified by peer review) is the author/funder. All rights reserved. No reuse allowed without permission.
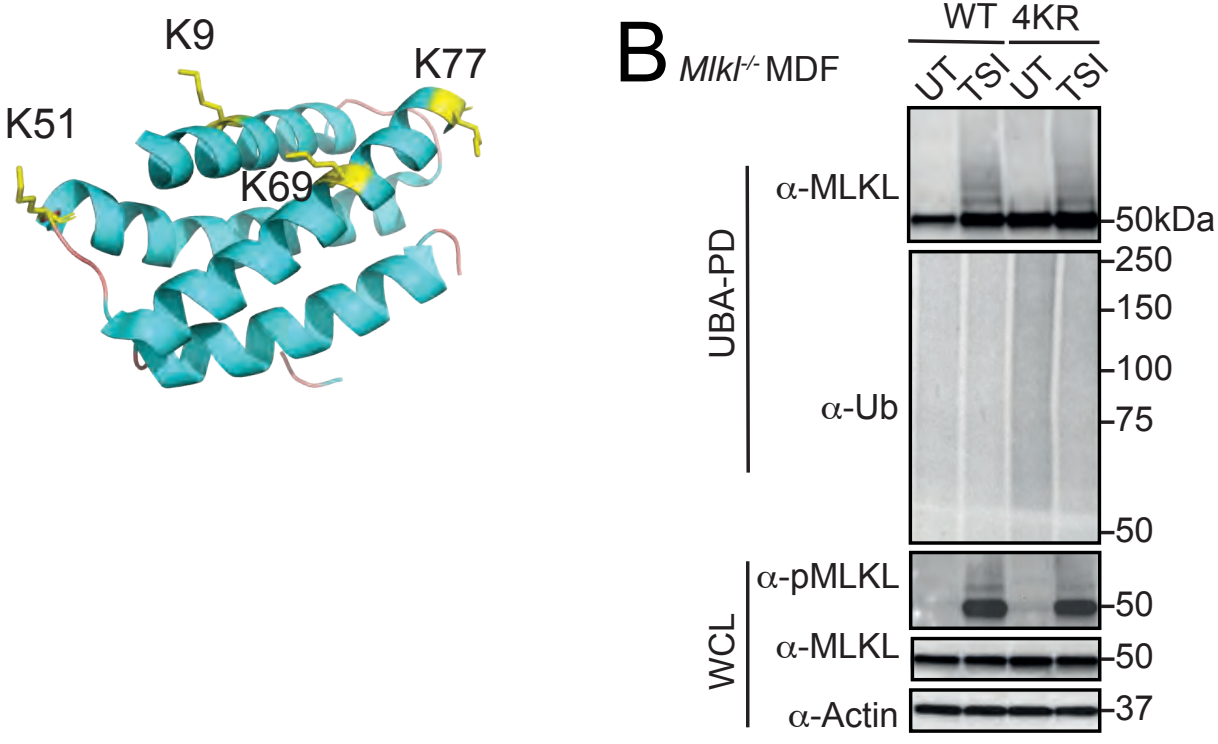
Supplementary Figure 6. Simultaneous arginine replacement of 4 ubiquitylation sites on the mouse MLKL 4HB domain

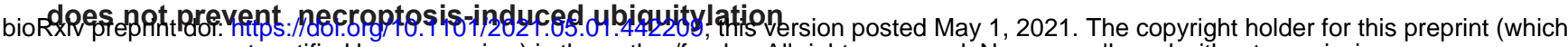

A

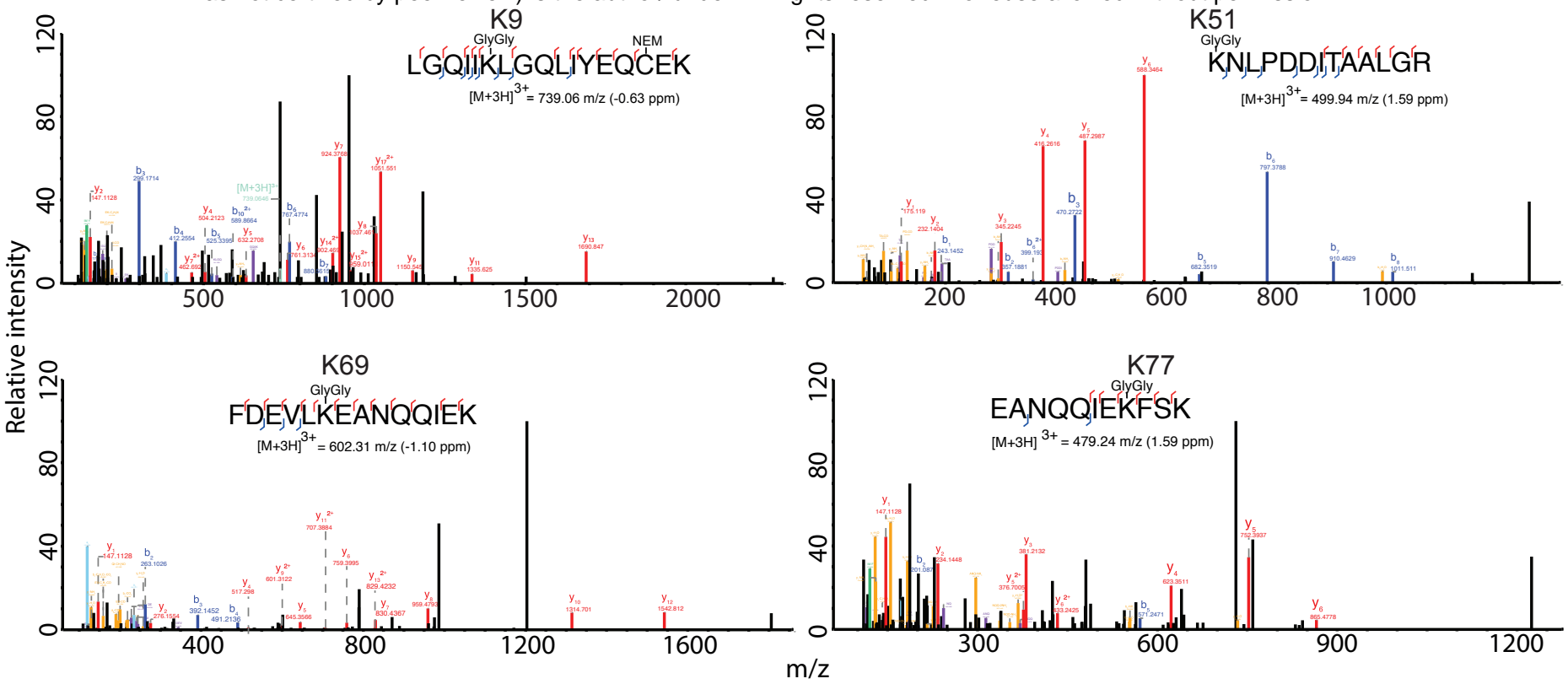

\section{B}

Mouse helix D2-E19

Helix C24-Q48

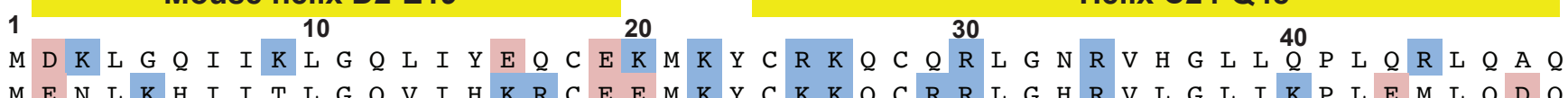

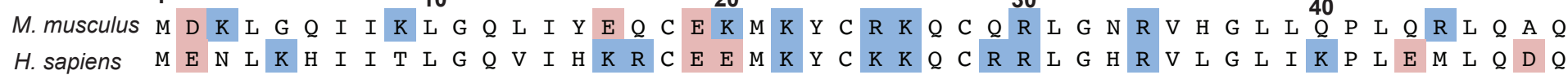

Helix P54-F78

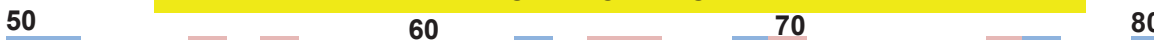

80

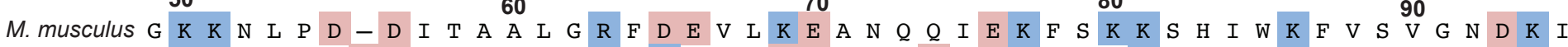

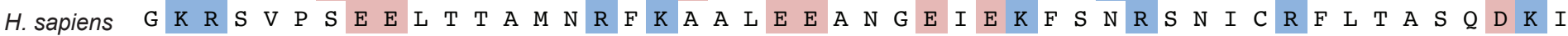

\section{Helix F97-L115}

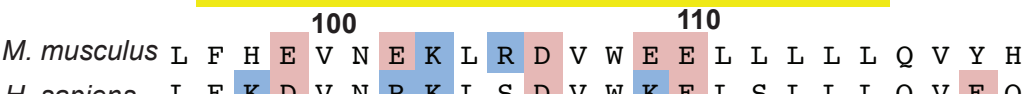

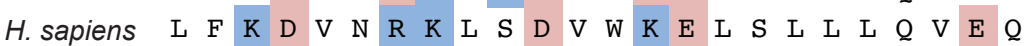

Mlkt-- MDF

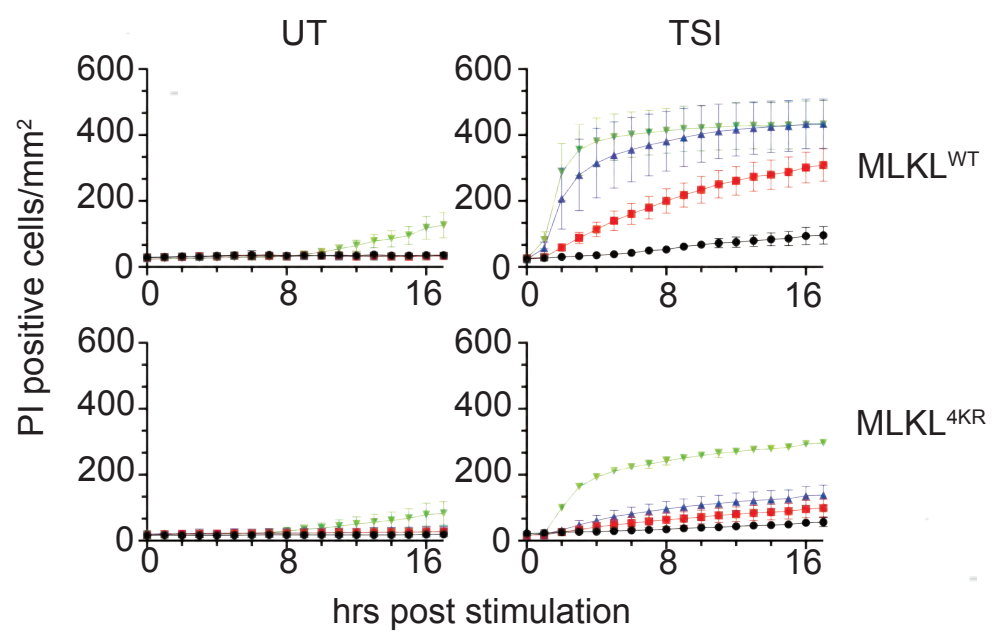

- $\quad 0 \mathrm{ng} / \mathrm{mL}$ dox

- $10 \mathrm{ng} / \mathrm{mL}$ dox

- $20 \mathrm{ng} / \mathrm{mL}$ dox

> $100 \mathrm{ng} / \mathrm{mL}$ dox 
Figure 7. MLKL ubiquitylation antagonises necroptosis

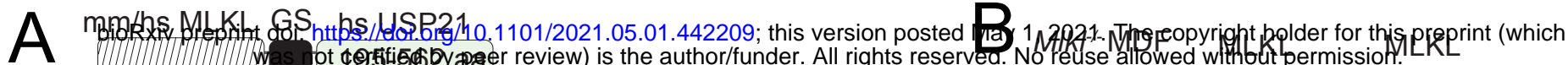
$\mathrm{mm} / \mathrm{hs}$ MLKL GS hs USP21 C221R

C

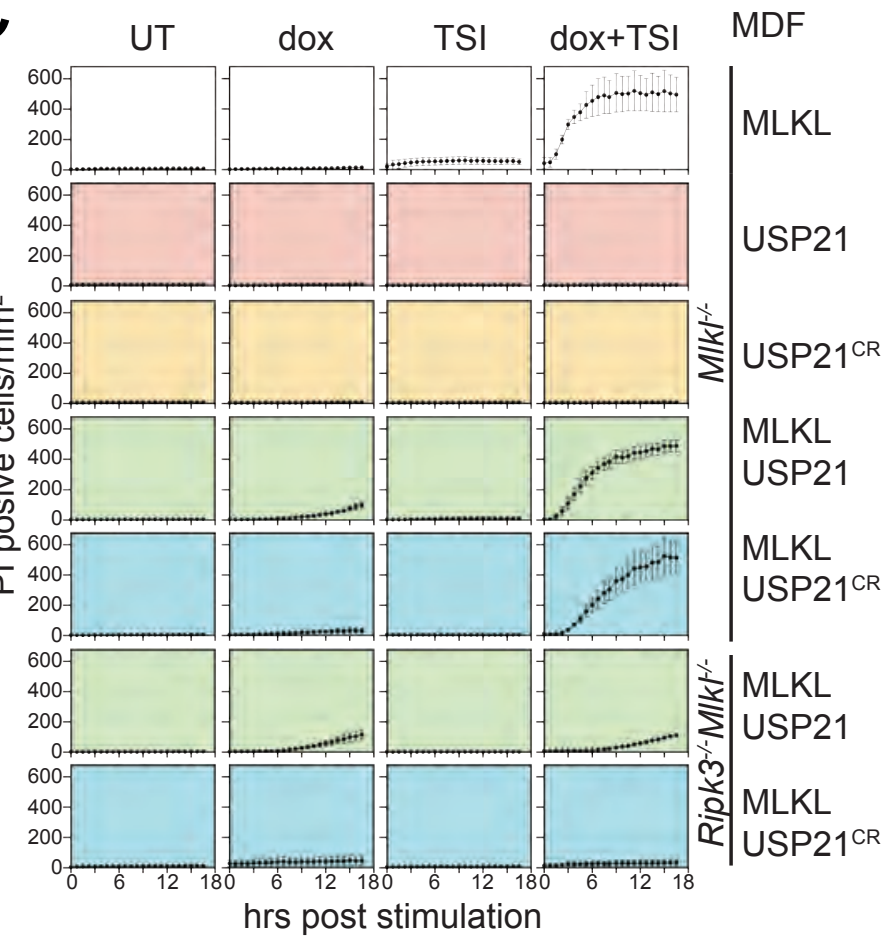
TSI hrs $\quad 0 \quad 2 \quad 2 \quad 4 \quad 6 \quad 0 \quad 2 \quad 2 \quad 4 \quad 6$

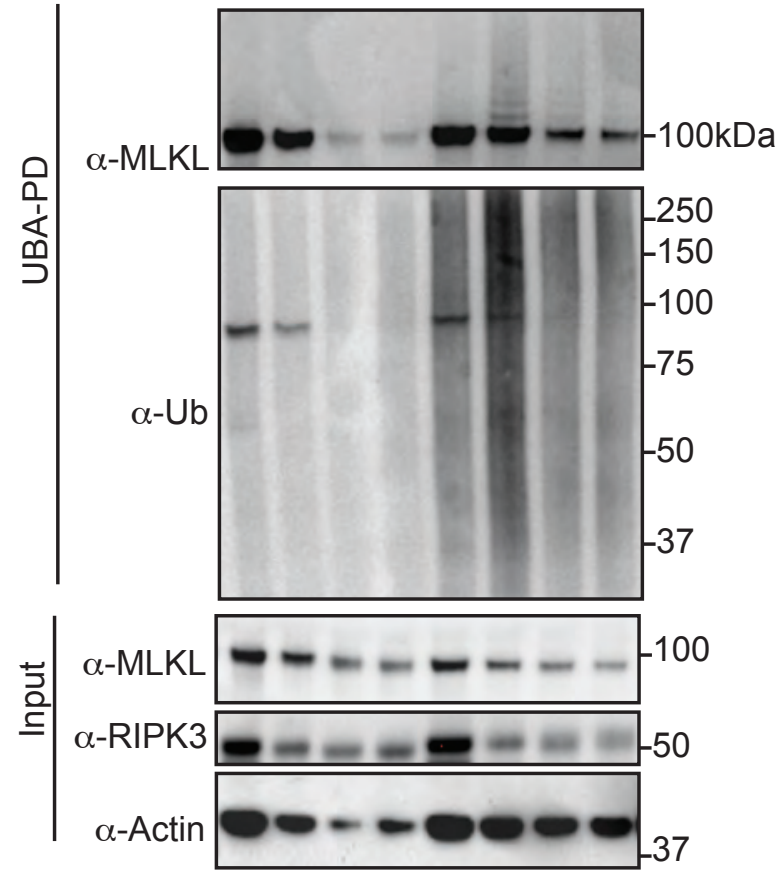

$\mathrm{D}$

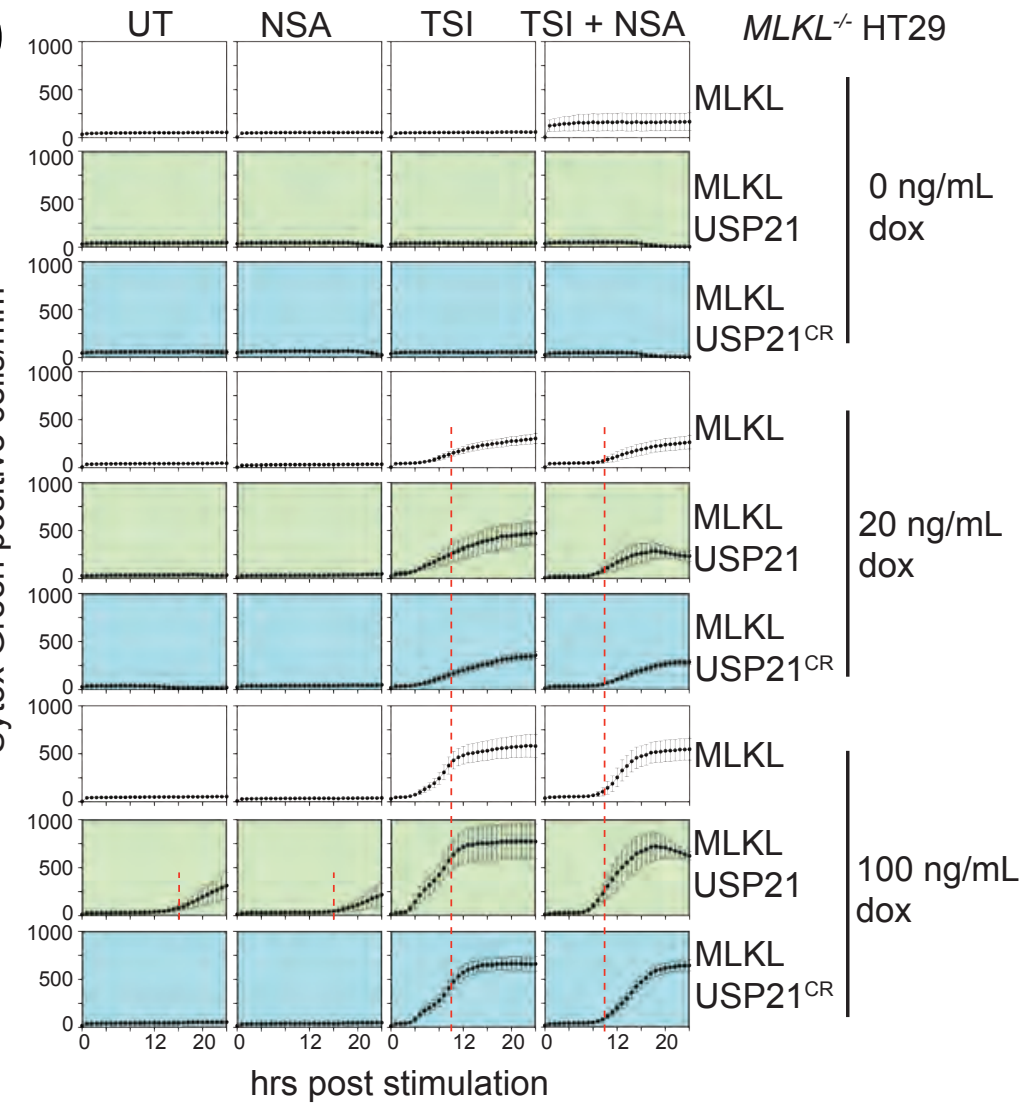

$\mathrm{E}$

$M L K L$ - $\mathrm{HT} 29 \mathrm{MLKL} \quad \mathrm{MLKL}$ USP21 USP21 1 CR
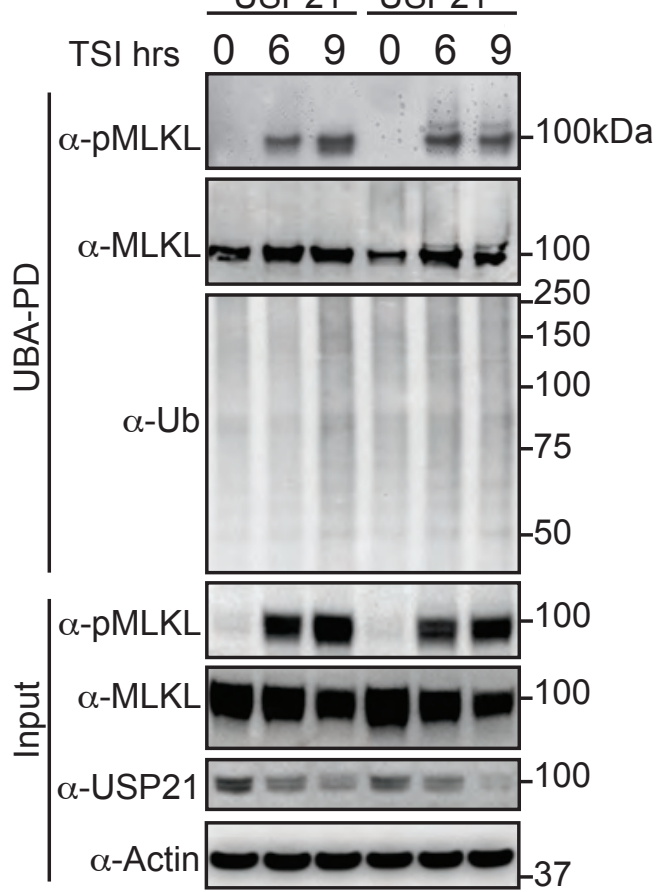
